Decision Support

\title{
Robust equilibria in location games
}

\author{
Berno Buechel ${ }^{\mathrm{a}}$, Nils Roehl ${ }^{\mathrm{b}, \mathrm{c}, *}$ \\ ${ }^{a}$ Department of Economics, University of Hamburg, Von-Melle-Park 5, 20146 Hamburg, Germany \\ ${ }^{\mathrm{b}}$ Department of Economics, University of Paderborn, Warburger Straße 100, 33098 Paderborn, Germany \\ c BiGSEM, Bielefeld University, Universitätsstraße 25, 33615 Bielefeld, Germany
}

\section{A R T I C L E I N F O}

Article history:

Received 16 August 2013

Accepted 17 July 2014

Available online 30 July 2014

\section{Keywords:}

Location

Spatial competition

Game theory

Networks

Minimal differentiation

\begin{abstract}
A B S T R A C T
In the framework of spatial competition, two or more players strategically choose a location in order to attract consumers. It is assumed standardly that consumers with the same favorite location fully agree on the ranking of all possible locations. To investigate the necessity of this questionable and restrictive assumption, we model heterogeneity in consumers' distance perceptions by individual edge lengths of a given graph. A profile of location choices is called a "robust equilibrium" if it is a Nash equilibrium in several games which differ only by the consumers' perceptions of distances. For a finite number of players and any distribution of consumers, we provide a complete characterization of robust equilibria and derive structural conditions for their existence. Furthermore, we discuss whether the classical observations of minimal differentiation and inefficiency are robust phenomena. Thereby, we find strong support for an old conjecture that in equilibrium firms form local clusters.
\end{abstract}

(c) 2014 Elsevier B.V. All rights reserved.

\section{Introduction}

In his classic example, Harold Hotelling illustrates competition in a heterogeneous market by two firms that consider where to place their shop on a main street (Hotelling, 1929). Ever since, this model of spatial competition has inspired a tremendous amount of research in various disciplines. Starting with Downs (1957), it is used to analyze the positioning of political candidates competing for voters (e.g., Mueller, 2003; Roemer, 2001) and to analyze the positioning of products in order to attract consumers (e.g., Carpenter, 1989; Salop, 1979). In the year 2013 alone, Hotelling has been cited more than 450 and Downs even more than 1100 times. ${ }^{1}$ Moreover, the model implication of minimal differentiation is known far beyond scholarly circles. In this paper, we want to challenge a fundamental aspect of the Hotelling-Downs approach.

Throughout the literature (of spatial competition), it has been virtually always assumed that consumers or voters who prefer the same position fully agree upon the ranking of the other alternatives, i.e., they have identical preferences or utility functions. This very strong homogeneity requirement can be considered as driven by the assumption that all consumers/voters use the same distance measure since in the standard Hotelling-Downs set-up

\footnotetext{
* Corresponding author at: Department of Economics, University of Paderborn, Warburger Straße 100, 33098 Paderborn, Germany. Tel.: +49 (0)5251 603889.

E-mail addresses: Berno.Buechel@wiso.uni-hamburg.de (B. Buechel), nils.roehl@ uni-paderborn.de (N. Roehl).

1 Google Scholar, February 10, 2014.
}

(dis)utility is represented by the distance between positions. In particular, if two people prefer the same option, in any spatial representation with homogeneous distances they necessarily rank all the other alternatives in the same order. This is hard to justify when we think of voters of the same political party who disagree about the second-best party, or of consumers with the same favorite brand but disagreement about the ordering of two other brands. And even in the case of geographic location choices the requirement appears to be challengeable if the distances represent travel time, for instance. ${ }^{2}$ As a matter of fact, these simple cases already exceed the scope of almost any model of locational competition.

Consider, for example, a poll on a group of voters about their favorite tax rate. The answers can be displayed as locations on a line. Location games that capture this application consider classically two political candidates who strategically choose a tax rate which they propose to the voters. Thereby it is standardly assumed that (a) each voter casts his vote for the candidate that is closest to him and (b) all voters asses the distances between the candidates homogeneously. In combination these two assumptions are not at all innocuous. As indicated above, they hide the homogeneity requirement that all voters who consider a tax rate of $10 \%$, for instance, as their favorite alternative, are supposed to rank any two tax rates, like $2 \%$ and $20 \%$, for example, in exactly the same

\footnotetext{
2 Indeed, it is possible that two individuals differ in their speed of walking uphill such that they would not choose the same path although both easily agree that there is one short and steep path and one longer and flatter path.
} 
order. Since this requirement is unnaturally strong, the classical result that two vote-maximizing candidates choose the median location (Hotelling, 1929) stands apparently on highly questionable grounds. A way to avoid this issue would be to ask the participants in the poll not only about their favorite tax rate, but about a full ranking of the alternative tax rates. Apart from practical problems, the downside of such an approach is the informational requirement that political candidates know the full assessment of every voter. That is, we have replaced a questionable requirement by another one. A solution to this issue relates back to the seminal contribution of Black (1948). He examined single-peaked preferences on a line, which has the same effect as voters who are allowed to asses the "distances" between different tax rates individually. Black's result that under single-peaked preferences the median voter wins in majority voting against any other alternative has the following implication for the situation of spatial competition outlined above: in any location game that is consistent with the poll, both candidates choose the median tax rate in equilibrium. In that sense the classical result is robust.

The example on tax rates illustrates that in two-player location games on a line the questionable requirement of homogeneous distance perceptions is not driving the final outcome. However, for all other cases - in particular, for more than two players and for multi-dimensional spaces - robustness of the results is an open problem. If one can show that the model assumption is not driving the results, then the model is put on a solid foundation. This issue, although fundamental, seems to have been overlooked in the - rich and exciting - history of location games.

In this paper we want to scrutinize for given outcomes of spatial competition whether they rely on homogeneous distance perceptions or not. To this end, we formalize individual distance perceptions as individual edge lengths of a graph. ${ }^{3} \mathrm{~A}$ formal description of consumers/voters of this type leads to a non-cooperative game between $p$ players, which are interpreted as firms or political candidates. In this game, players simultaneously choose a location in order to maximize the number of agents (i.e., consumers/voters) they can attract. An equilibrium is then called robust if it is an equilibrium for all possible distance perceptions that are based on the same underlying structure (a line, for example). In other words, our modeling approach boils down to defining a stronger notion of equilibrium which we call robust equilibrium. It is defined directly on the situation of spatial competition, i.e., the underlying space and the distribution of agents (such as the poll on tax rates). Formally, several of location games correspond to the same situation of spatial competition, one for each setting of individual distance perceptions; and a robust equilibrium is a Nash equilibrium in any of these games. In particular, it is also a Nash equilibrium in the standard case of homogeneous distances.

A key result for our analysis is the characterization of robust equilibria by four conditions which are jointly necessary and sufficient. It is based on partitioning the underlying space into "hinterlands" and "competitive zones". Applying this result allows us first of all to judge which of the standard results are robust. In fact, we find that several outcomes do not depend on the assumption of homogeneous distances, but others do.

In the second part of the paper, we examine general properties of robust equilibria. Among them is the central issue of minimal differentiation (e.g., d'Aspremont, Gabszewicz, \& Thisse, 1979; de

\footnotetext{
${ }^{3}$ This can be shown to be equivalent to the assumption of single-peaked preferences on certain domains. For example, if the underlying structure is a line graph, then this assumption is equivalent to the standard notion of single-peakedness. An alternative model variation would keep the assumption of homogenous distances but add a set of nodes (which we call "dummy nodes") to make the graph more flexible. As we show in Appendix B, this model variation would undermine the model's explanatory power.
}

Palma, Ginsburgh, Papageorgiou, \& Thisse, 1985; de Palma, Hong, \& Thisse, 1990; Eaton \& Lipsey, 1975; Economides, 1986; Król, 2012; Meagher \& Zauner, 2004). It turns out that robust equilibria satisfy a local variant of minimal differentiation, i.e., they induce reduced games in which the corresponding players are minimally differentiated. This result provides strong support for the "principle of minimal clustering" which has been proposed in the seminal contribution of Eaton and Lipsey (1975). Indeed, for any number of players, any underlying structure, and any distribution of agents, robust equilibria are characterized by clusters of players. That is, the players are jointly located on what we show to be the appropriately defined medians of local areas. Based on this result, we discuss the welfare implications for consumers and observe that almost all robust equilibria are not Pareto efficient. Consumers would unambiguously improve if some firm would be relocated appropriately. We finally, elaborate on the conditions for the existence of robust equilibria. We analyze how the spatial structure and the distribution of consumers/voters guarantee, admit, or preclude the existence of robust equilibria. Interestingly, two very common assumptions in the literature - (a) uniform distribution of consumers/voters and (b) one-dimensional space such as cycle or line structures - are mutually exclusive in the sense that for higher numbers of players robust equilibria require that one of them is not satisfied.

\subsection{Related literature}

There is an immense body of literature on spatial competition. While the original Hotelling-Downs framework is restricted to a one-dimensional space, a uniform distribution of agents, and only two players, many authors have attempted to relax these restrictions. To do so, one branch of the literature has followed a continuous modeling approach within the Euclidean space $\mathbb{R}^{k}$ (e.g., d'Aspremont et al., 1979; Economides, 1986), while a second branch replaces the Euclidean space by a graph (e.g., Labbé \& Hakimi, 1991). Because the history of both branches is rich and long, providing a summary which covers all of it would exceed the scope of our paper. We restrict ourselves here to list several surveys on the topic and to discuss the most closely related works.

A broad overview and taxonomy of literature on spatial competition can be found in Eiselt, Laporte, and Thisse (1993). Based on five components (the underlying space, the number of players, the pricing policy, the rules of the game, and the behavior of the agents) the authors provide a bibliography for competitive location models. While this summary is not limited to certain subbranches, more specific surveys have been written on spatial models of consumer product spaces (Lancaster, 1990), on spatial competition in continuous space (Gabszewicz \& Thisse, 1992), on spatial models of political competition (Mueller, 2003; Osborne, 1995), on competition in discrete location models (Plastria, 2001), on sequential competition (Eiselt \& Laporte, 1997; Kress \& Pesch, 2012), and on one-stage competition in location models (Eiselt \& Marianov, 2011; ReVelle \& Eiselt, 2005).

Although there are many variations and relaxations of spatial competition, virtually all of the models rely on the assumption of homogeneous distance perceptions. For instance, asymmetric transportation costs (e.g., Nilssen, 1997) do not alter the assumption. In order to examine to which extent this standard simplification is driving the results we will focus on the first stage of Hotelling's game, i.e., we will investigate the location choices of the players but we will not include additional variables such as prices. Similar approaches have been used, for example, by Eaton and Lipsey (1975), Denzau, Kats, and Slutsky (1985), and Braid (2005) who also concentrate on spatial competition by assuming fixed (and equal) prices. Nevertheless, extending our approach to a two-stage game would be a potential next step for further 
research. Integrating heterogeneous consumer behavior into a model of spatial competition has been attempted by a few studies only. Among them are de Palma et al. (1985, 1990) and Rhee (1996) who find that ambiguity about consumers' (or voters') behavior may lead to minimal differentiation. More specifically, they show that if the consumers' preferences do not only depend on prices and distances but also on inherent product characteristics and, furthermore, the firms have incomplete information about consumers' tastes, then Hotelling's main result can be restored under certain conditions. This conclusion is not confirmed in closely related models where the authors assume that the exact position of demand is unknown (e.g. Król, 2012; Meagher \& Zauner, 2004, 2005). Thus, the validity of minimal differentiation under heterogeneous agents is still an open problem and the same holds true for the main implications, for example, that spatial competition generically does not lead to socially efficient outcomes. However, the previously cited publications differ from our work in at least two important aspects. First, in these works, players are assumed to have a probability distribution for the behavior of agents. In our work, uncertainty is not explicitly modeled but only enters implicitly as robust equilibria do not depend on specification details about the agents' behavior. Second, the way we model and interpret heterogeneity differs from the approaches of the other authors. In our setting, the agents apply individual distances to compare specific product variations but the preferences do not depend on inherent product characteristics. To model this in a convenient way we use a graph-based approach. We believe that our definitions are more intuitive in discrete spaces than in the plane and that this approach helps to highlight the difference between homogeneous and heterogeneous agents. However, the main questions of our work are not restricted to graphs and thus our contribution should also be interesting in a more general context. To the best of our knowledge, this is the first paper that assesses robustness of equilibria in location games with respect to different distance perceptions.

From a technical point of view, the model of Eiselt and Laporte $(1991,1993)$ is heavily related to ours. In these publications, the authors show for homogeneous agents that the two-player and three-player cases on trees always result in some kind of minimal differentiation. We will check whether this is also true in our more general context of more than two players and arbitrary graphs. More recently, Shiode and Drezner (2003) studied the two-player case on trees under sequential location choices and stochastic demand. Further recent contributions, to name but a few, deal with terrorism (e.g., Berman \& Gavious, 2007) or stem from computer science (e.g., Godinho \& Dias, 2010; Jiang, Leyton-Brown, \& Bhat, 2011; Mavronicolas, Monien, Papadopoulou, \& Schoppmann, 2008). Still, the issue of heterogeneous distances is not addressed in these publications.

\section{The model}

Our modeling approach proceeds in two steps. First we consider, as usual, a non-cooperative game between players (the firms/candidates) who are able to occupy a position or object. The agents (consumers/voters) are still attracted by the player(s) located closest to them but now their distance perceptions may be assessed on an individual basis. More specifically, the agents agree on the underlying space which is modeled by means of a graph (Section 2.1), but in our setting they may individually measure the similarity between the objects (Section 2.2). Then, in the second step, we study whether equilibria of the game are robust with respect to perturbations of the distance perceptions. To this end, roughly speaking, we fully abandon the distances. This means formally that an outcome is called robust if it is an equilibrium for

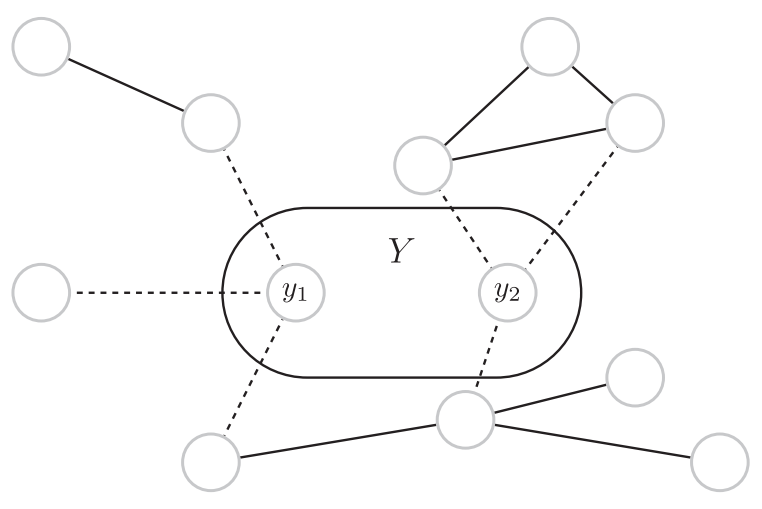

Fig. 1. Deletion of nodes.

all possible edge lengths of the same underlying graph (Section 2.3). If this is satisfied, the outcome is completely independent of individual distance perceptions and then the standard case of homogeneous distances is a well-justified simplification.

\subsection{Definitions of graphs}

An undirected graph $(X, E)$ consists of a set of vertices or nodes $X$ and a set of edges $E$ where each edge is a subset of the vertices of size two. Let $X$ be a finite set of size $\xi \geqslant 2$. For brevity, we write $x y$ or $y x$ for an edge $\{x, y\} \in E$. Given a graph $(X, E)$, we denote by $N_{x}:=\{y \in X \mid x y \in E\}$ the set of neighbors of a node $x$. The number of edges/neighbors is its degree $\operatorname{deg}_{x}:=\left|N_{x}\right|$. Furthermore, $Y \subseteq X \backslash\{x\}$ is neighboring to $x \in X$ if there exists some $y \in Y$ with $x y \in E$.

A path from $x \in X$ to $x^{\prime} \in X$ in $(X, E)$ is a sequence of distinct nodes $\left(x_{1}, \ldots, x_{T}\right)$ such that $x_{1}=x, x_{T}=x^{\prime}$, and $x_{t} x_{t+1} \in E$ for all $t \in\{1, \ldots, T-1\}$. A set of nodes $Y \subseteq X$ is said to be connected if for any pair $y, y^{\prime} \in Y$ there exists a path between the two nodes. A set of connected nodes is called a component if there is no path to nodes outside of this set, i.e., $C \subseteq X$ is a component of $(X, E)$ if it is connected and for all $x, x^{\prime}$ such that $x \in C$ and $x^{\prime} \in X \backslash C$ there does not exist any path. A graph that consists of only one component is called connected because then there is a path between any two nodes. Throughout the paper, we will restrict attention to connected graphs. An important class of such graphs is the class of trees. Trees are connected with $\xi-1$ edges or, equivalently, in a tree each pair of vertices is connected by a unique path.

A node-weighted graph is a triple $(X, E, w)$, where $w:=$ $\left(w_{x}\right)_{x \in X} \in \mathbb{R}_{+}^{\xi}$ is a vector of weights. We write $w_{x}$ for the weight of node $x \in X$ and $w(Y)=\sum_{y \in Y} w_{y}$ for the weight of a set of nodes $Y \subseteq X$. The weight $w$ will be determined later on by the distribution of agents.

Now, let $(X, E, w)$ be given. An important operation in graphs is to delete a set of nodes $Y \subseteq X$ and all involved edges: $(X, E)-Y:=\left(X \backslash Y, E_{\mid X \backslash Y}\right)$ with $E_{\mid X \backslash Y}=\{x y \in E \mid x, y \in X \backslash Y\}$. This is illustrated in Fig. 1.

The operation $(X, E)-Y$ leads to a graph with potentially several components and we denote them by $C_{1}^{Y}, C_{2}^{Y}, \cdots, C_{l_{Y}}^{Y}$ such that $w\left(C_{1}^{Y}\right) \geqslant w\left(C_{2}^{Y}\right) \geqslant \cdots \geqslant w\left(C_{l_{Y}}^{Y}\right)$. If $l_{Y}>1$ and $|Y|=1$, say $Y=\{x\}$, the node is called a cut vertex (cf., e.g., Diestel, 2005) and we write $C_{k}^{x}$ instead of $C_{k}^{\{x\}}$. In this case, for the number of components it holds that it is not greater than the degree of $x$. A connected set of nodes $B \subseteq X$ is called a block if there is no cut vertex in $(X, E)-X \backslash B=\left(B, E_{\mid B}\right)$ and $B$ is maximal with respect to inclusion, i.e., $B \subsetneq B^{\prime} \subseteq X$ implies that there exists a cut vertex in $\left(B^{\prime}, E_{\mid B^{\prime}}\right)$. That is, a set of nodes is a block if the induced subgraph cannot be decomposed into multiple components by deleting single nodes and it is not possible to find a larger subgraph with this feature. 
Note that $x \in X$ is contained in several blocks if and only if it is a cut vertex. The set of blocks of a given graph is denoted by $\mathcal{B}$ and $b:=|\mathcal{B}|$ is the number of blocks.

\subsection{Perceived distances and players' payoffs}

In the following, the elements of $X$ are called objects and are interpreted, according to the three applications, as geographical locations, political platforms or product specifications. Let $N=\left\{i_{1}, \ldots, i_{n}\right\}$ be a finite set of agents who have a favorite object $\hat{\chi}^{i} \in X$. As usual, the graph $(X, E)$ is used to represent the relations between the objects as they are perceived by the agents. ${ }^{4}$ In order to be as general as possible we impose no further requirements on the structure of the graph, but typical examples from literature are lines, cycles or lattices, to name but a few. In contrast to previous works, we assume that perceptions are subjective to some extent. Formally, for each $i \in N$ there are edge lengths $\left(\delta_{e}^{i}\right)_{e \in E}>0$ that represent his individual estimation of distances between the nodes, such that, for example, $\delta_{e}^{i}$ need not coincide with $\delta_{e}^{j}{ }^{5}$ Given $\delta:=\left(\delta_{e}^{i}\right)_{e \in E^{\prime}}^{i \in N}$, agent $i$ 's perceived distance $d^{i}(x)$ to an object $x \in X$ is the length of the shortest path(s) from the favorite object $\hat{x}^{i}$ to $x$, where the length of a path is the sum of its edge lengths:

$d^{i}(x):=\min \left\{\sum_{t=1}^{T-1} \delta_{x_{t} x_{t+1}}^{i} \mid\left(x_{1}, \ldots, x_{T}\right)\right.$ is a path from $\hat{x}^{i}$ to $\left.x\right\}$.

We set $d^{i}\left(\hat{x}^{i}\right)=0$ for all $i \in N$. Note that two agents with the same favorite object, i.e., $\hat{x}^{i}=\hat{x}^{j}$, might have different perceptions about the distances to the other objects. As usual, we will assume a "distance-based behavior" of the agents, i.e., agent $i \in N$ weakly prefers an object $x \in X$ over $y \in X$ if and only if $d^{i}(x) \leqslant d^{i}(y)$. In other words: his utility is decreasing in distances. Thus, the preferences of agent $i \in N$ are completely determined by his favorite object $\hat{x}^{i}$ and his individual edge lengths $\left(\delta_{e}^{i}\right)_{e \in E}{ }^{6}$ With the assumption that $\delta_{e}^{i}=\delta_{e}^{j}$ for all $i, j \in N$ and any $e \in E$, we obtain the standard model, where distance perceptions are homogeneous.

In addition to the objects and agents, we consider a set of players $P:=\left\{c_{1}, \ldots, c_{p}\right\}$ of finite size $p \geqslant 2$. To ease the distinction between agents and players we will use the male form for agents, while players are assumed to be female. Each $c \in P$ is supposed to occupy an object $x \in X$. Formally, the strategy set for each player $c \in P$ is $S^{c}=X$, such that a strategy is an object $s^{c} \in X$. Let $S=S^{c_{1}} \times \cdots \times S^{c_{p}}$. Given a strategy profile $s \in S$, let $p_{x} \in \mathbb{N}$ be the number of players whose strategy is $x \in X$. Furthermore, let $\Phi^{i}(s)$ be the set of players who are perceived as closest by agent $i \in N$, i.e., $\Phi^{i}(s)=\left\{c \in P \mid d^{i}\left(s^{c}\right) \leqslant d^{i}\left(s^{\bar{c}}\right) \forall \bar{c} \in P\right\}$. Note that we loosely

\footnotetext{
${ }^{4}$ For reasons discussed in Appendix B, we do not allow for "dummy nodes," that is, we do not consider the possibility of adding further nodes to the graph which are not objects.

${ }^{5}$ The interpretation for geographic locations is as follows: the agents agree on the underlying graph (a road map, for example) but they are heterogeneous in terms of assessing or evaluating the edge lengths (the travel time, for example). If the graph does not represent geographic distances, but policy spaces or the perception of brands, it seems to be an even more unrealistic assumption that all agents use the same distance measure, as motivated in the introduction.

${ }^{6}$ There is a justification for this type of preference which neither deals with differing edge lengths nor with distance-based behavior. Agents can be assumed to have single-peaked preferences on the graph as they were defined for lines (Black, 1948) or trees (Demange, 1982). Such preferences find broad acceptance and play a crucial role in the literature on social choice (see, e.g., Moulin, 1980). The alternative formulation with single-peaked preferences is, in fact, equivalent to the (quite different) formulation here. The proof for this claim can be requested from the authors.
}

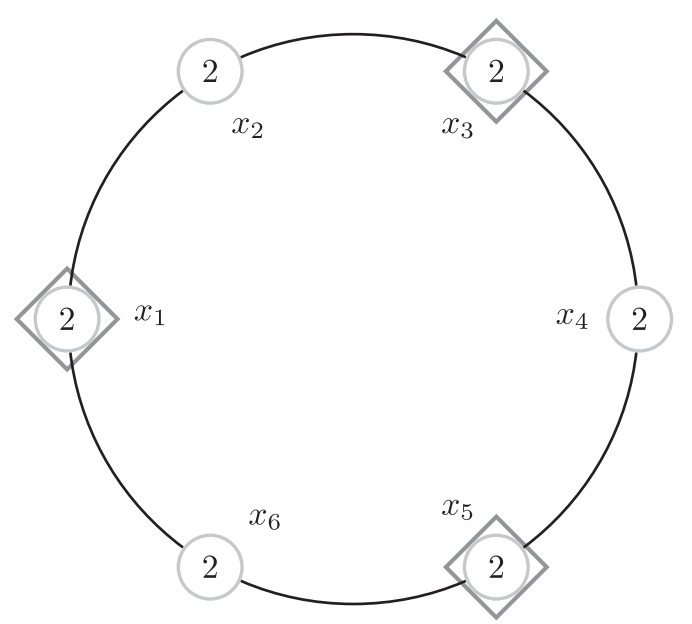

Fig. 2. Three players on a cycle graph.

speak about the perceived distance to a player $c \in P$ instead of the distance to the player's chosen object $s^{c} \in X$. We assume that each agent is allocated to the player which is perceived as closest. If multiple players are perceived as closest by some agent, then he is assumed to be uniformly distributed among these players. Thus, given a strategy profile $s \in S$, player c's payoff $\Phi^{c}(s)$ is the mass of agents who perceive object $s^{c}$ as closest to their favorite object, i.e., the payoff of $c \in P$ is given by $\pi^{c}(s)=\sum_{i: c \in \Phi^{i}(s)} \frac{1}{\left|\Phi^{i}(s)\right|}$. A profile of payoffs is denoted by $\pi_{\delta}:=\left(\pi_{\delta}^{c}\right)^{c \in P}:=\left(\pi^{c}\right)^{c \in P}$, where the subscript $\delta$ indicates that the payoffs depend on the individual edge lengths $\delta=\left(\delta_{e}^{i}\right)_{e \in E}^{i \in N}$.

\subsection{Equilibrium notions}

Fix a graph $(X, E)$ and a set of agents $N$ such that for each agent $i \in N$ we have a favorite object $\hat{x}^{i} \in X$ and individually measured edge lengths $\left(\delta_{e}^{i}\right)_{e \in E}$. Then a normal form game is given by $\Gamma^{\delta}=\left(P, S, \pi_{\delta}\right)$. The game is indexed by $\delta$ to emphasize that the payoffs, and therefore the game depends on the individual edge lengths. The main goal of our work is to examine to which extent this restriction determines the outcome of the standard setting, which is the special case of homogeneous distances. A Nash equilibrium of the game $\Gamma^{\delta}$ is also called a locational (Nash) equilibrium (cf. Eiselt \& Laporte, 1991, 1993). Thus, $s \in S$ is a locational equilibrium if for all $c \in P$ and for all $x \in X$ we have $\pi^{c}\left(s^{c}, s^{-c}\right) \geqslant \pi^{c}\left(x, s^{-c}\right)$.

Example 1. Consider a cycle graph $(X, E)$ on six nodes, i.e., $X=\left\{x_{1}, x_{2}, \ldots, x_{6}\right\}$ and $E=\left\{x_{1} x_{2}, x_{2} x_{3}, \ldots, x_{6} x_{1}\right\}$. Let $N=\left\{i_{1}\right.$, $\left.i_{2}, \ldots, i_{12}\right\}$ be a set of twelve agents with favorite objects $\left(\hat{x}^{1}, \hat{x}^{2}, \ldots, \hat{x}^{12}\right)=\left(x_{1}, x_{1}, x_{2}, x_{2}, \ldots, x_{6}, x_{6}\right)$. We first assume homogeneous edge lengths, i.e., for all $i \in N$ we have $\delta_{e}^{i}=1$ for any $e \in E$. Together with a set of three players $P=\left\{c_{1}, c_{2}, c_{3}\right\}$ this constitutes a game $\Gamma^{\delta}$.

The graph $(X, E)$ is illustrated in Fig. 2. The number within a node indicates the number of agents who have this node as the favorite object. The edge lengths are not represented. Finally, the three squares represent the strategy profile $\left(s^{1}, s^{2}, s^{3}\right)=\left(x_{1}, x_{3}, x_{5}\right)$. We will keep these conventions in the following figures.

For this game, results of Mavronicolas et al. (2008) imply that the depicted strategy profile $s$ is a locational equilibrium. A player cannot improve by relocating, because her payoff either remains 4 (when deviating to a neighbor) or decreases. This result, however, 
depends on the specific edge lengths. Consider the situation where one of the two agents with favorite object on $x_{2}$ assigns a different length to an edge next to him, such as $\tilde{\delta}_{x_{1} x_{2}}^{3}=1-\epsilon$ for some $\epsilon>0$ and $\tilde{\delta}_{e}^{3}=1$ for all other edges. The perceived distances of the other agents are assumed to stay the same. Then the depicted strategy profile $s$ is not a locational equilibrium. The player $c_{3} \in P$ with strategy $x_{3}$ now has an incentive to deviate to $x_{2}$ or $x_{4}$ because in both cases she would attract four agents instead of only 3.5. Thus, the strategy profile $s \in S$ is a locational equilibrium in the game $\Gamma^{\delta}$ but not in the perturbed game $\Gamma^{\tilde{\delta}}$. In some sense the profile is not "robust".

The previous example motivates the following definition.

Definition 1 (Robust equilibrium). A strategy profile $s^{*} \in S$ is a robust equilibrium if it is a locational equilibrium for any collection of individual edge lengths. In other words: $s^{*} \in S$ is a locational equilibrium in $\Gamma^{\delta}$ for any $\delta=\left(\delta_{e}^{i}\right)_{e \in E}^{i \in N}$.

Certainly, robustness is a strong requirement. But it is a desirable property for at least two reasons. First, a robust equilibrium is independent of the assumption of homogeneous edge lengths but includes this as a special case. Indeed, a robust equilibrium is also a locational equilibrium in the homogeneous case $\Gamma^{\delta}$, where $\left(\delta_{e}^{i}\right)_{e \in E}$ is the same for all agents $i \in N$. Second, to determine the locational equilibrium one has to specify for each agent her favorite object $\hat{\chi}^{i} \in X$ as well as her list of edge lengths $\left(\delta_{e}^{i}\right)_{e \in E}$ together with a graph $(X, E)$. On the other hand, to determine robust equilibria it is sufficient to know the graph $(X, E)$ and the distribution of favorite objects $\left(\hat{x}^{i}\right)^{i \in N}$. In fact, it is sufficient to have only information about the node-weighted graph that is induced by $\left(\hat{x}^{i}\right)^{i \in N}$, i.e., it is enough to know $(X, E, w)$ where $w_{x}:=\left|\left\{i \in N \mid \hat{x}^{i}=x\right\}\right|$ is the number of agents having $x$ as their favorite object. We will interpret an exogenously given node-weighted graph $(X, E, w)$ as a situation of spatial competition.

\section{Robustness}

We will first give a characterization of robustness which applies to test whether locational equilibria are robust. Then, we will turn to properties of robust equilibria, in particular minimal differentiation and efficiency. Finally, we will reconsider the existence of robust equilibria.

\subsection{Characterization}

In this subsection we provide the necessary and sufficient conditions for a strategy profile to be a robust equilibrium. For this purpose we need additional definitions.

Definition 2. Let $(X, E)$ be a graph and fix a strategy profile $s \in S$. Furthermore, let $\bar{X}=\bigcup_{c=1}^{p}\left\{s^{c}\right\} \subseteq X$ be the set of occupied nodes in $s$.

- The hinterland $H_{x} \subseteq X$ of node $x \in \bar{X}$ is the set of nodes that have $x$ on every path to any $x^{\prime} \in \bar{X}$. In the special case where all players choose the same strategy (i.e., $|\bar{X}|=1$ ), say $\bar{X}=\{x\}$, we define $H_{x}:=X$.

- An unoccupied zone $Z \subseteq X$ is a component of $(X, E)-\bar{X}$. The set of all unoccupied zones is denoted by $\mathcal{Z}$.

- An unoccupied zone $Y \subseteq X$ is called a competitive zone if it is not contained in any hinterland, i.e., $Y \nsubseteq H_{x}$ for all $x \in \bar{X}$. The set of all competitive zones is $\mathcal{Y}$.

- Two distinct objects $x, x^{\prime} \in \bar{X}$ are indirectly neighboring if there exists a competitive zone to which both nodes are neighboring.

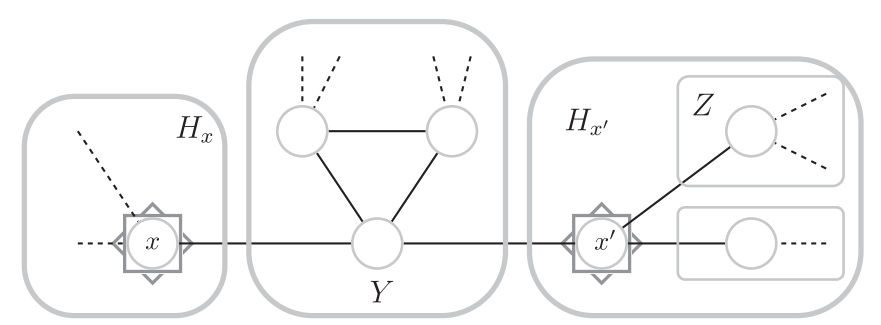

Fig. 3. Example for definitions: decomposition into competitive zones and hinterlands.

- The neighboring area $A_{x} \subseteq X$ of $x \in \bar{X}$ is the unoccupied zone which would be obtained when removing all players located on $x$. Formally, that is $A_{x}=\bigcup_{Z \in \mathcal{Z}_{x}} Z \cup\{x\}$, where $\mathcal{Z}_{x}:=\{Z \in$ $\mathcal{Z} \mid Z$ neighboring to $x\}$.

The notions of hinterland and competitive zone go back to Eiselt (1992) who has defined them for the positions of two players. The hinterland $H_{x} \subseteq X$ consists of the node itself and possibly several unoccupied zones that are adjacent to $x \in \bar{X}$ but not to any other occupied node in $\bar{X}$. Agents who have their favorite object in $H_{x}$ must be closer to player(s) on node $x$ than to all other players, since any path, and therefore also the shortest one(s), contain this object. This is different for competitive zones. Players surrounding a competitive zone $Y \in \mathcal{Y}$ compete with indirectly neighboring competitors over the agents who have their most favorite object in $Y$. The definitions are illustrated in Fig. 3, where there are two occupied nodes $x, x^{\prime} \in \bar{X}$, several unoccupied zones, where one of them $(Y)$ is a competitive zone, and another one $(Z)$ belongs to a hinterland. Furthermore, the neighboring area $A_{x} \subseteq X$ consists of the hinterland $H_{x}$ and the competitive zone $Y$, while the neighboring area $A_{x^{\prime}}$ consists of the other hinterland $H_{x^{\prime}}$ and the competitive zone $Y$. Generally, each node either belongs to one hinterland or to one competitive zone. This can be considered as a partition of $X$ into $l$ hinterlands (i.e., $|\bar{X}|=l$ ) and $k$ competitive zones

$\Pi(s)=\left\{H_{x_{1}}, \ldots, H_{x_{l}}, Y_{1}, \ldots, Y_{k}\right\}$.

In fact, because every agent with favorite object in $H_{x} \subseteq X$ is always closer to a player on the corresponding node $x$ than to any other occupied node, $\frac{w\left(H_{x}\right)}{p_{x}}$ is the "worst-case payoff" that a player who chooses $x$ receives (recall that $p_{x}$ is the number of players on node $x$ ). Conversely, the maximal payoff of a player who chooses $x$ is restricted by the neighboring area $A_{x} \subseteq X$, i.e., by $\frac{w\left(A_{x}\right)}{p_{x}}$. These simple considerations lead to the following key proposition.

Proposition 1. Let $s^{*} \in S$ be a strategy profile on a node-weighted graph $(X, E, w)$ and let $\Pi(s)$ be the corresponding partition as in (1). Furthermore, let $\widehat{Z} \in \operatorname{argmax}_{Z \in \mathcal{Z}} w(Z)$ be a heaviest unoccupied zone. Then $s^{*}$ is a robust equilibrium if and only if the following four conditions are satisfied for all $x \in \bar{X}$ :

(1.) $\frac{w\left(H_{x}\right)}{p_{x}} \geqslant w(\widehat{Z})$

(2.) $\frac{w\left(H_{x}\right)}{p_{x}} \geqslant \frac{w\left(A_{x^{\prime}}\right)}{p_{x^{\prime}}+1} \quad \forall x^{\prime} \in \bar{X} \backslash\{x\}$.

Furthermore, if $p_{x}=1$ :

(3.) $w(Y)=0 \quad \forall Y \in \mathcal{Y}, \quad Y \subseteq A_{x}$,

(4.) $w H_{x} \geqslant \frac{w\left(A_{x^{\prime}}\right)}{p_{x^{\prime}}} \quad \forall x^{\prime} \in \bar{X}$ (indirectly) neighbored to $x$.

Proof. We show first that the four conditions are jointly necessary. Then, in the second step, we turn to sufficiency. 


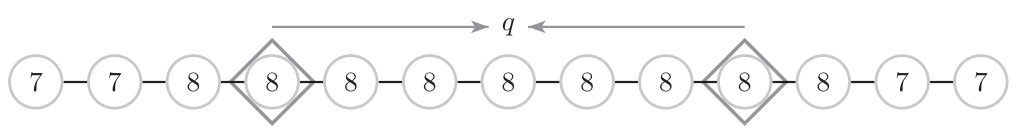

Fig. 4. A node-weighted line graph with two players.

Necessity: Assume $s^{*} \in S$ is a robust equilibrium with occupied nodes $\bar{X}=\left\{x_{1}, \ldots, x_{l}\right\}$ and assume $p_{x}=1$ where $x \in \bar{X}$. Let $c \in P$ be the player with $s^{c}=x$.

We first establish that $x$ is not neighboring a non-trivial competitive zone, i.e., $w(Y)=0$ for all $Y \in \mathcal{Y}$ neighboring to $x$. To see this suppose the opposite is true. Fix some arbitrary object $y \in Y$. Because $A_{x} \subseteq X$ is connected, it is possible to find edge lengths $\left(\bar{\delta}_{e}^{i}\right)_{e \in E}$ for all $i \in N$ with $\hat{x}^{i} \in A_{x} \backslash Y$ such that $d^{i}(y)<d^{i}\left(x^{\prime}\right)$ for any occupied position $x^{\prime} \in \bar{X}$ neighboring to $A_{x}$. This implies $d^{i}(x)<d^{i}(y)$ since every path in $A_{x}$ from $\hat{x}^{i}$ to $y$ passes through $x$. Furthermore, for all $j \in N$ with favorite object in $Y$ we can choose edge lengths $\left(\bar{\delta}_{e}^{i}\right)_{e \in E}$ such that $d^{j}(y)<d^{j}\left(x^{\prime \prime}\right)<d^{j} x$, where $x \neq x^{\prime \prime} \in \bar{X}$ is some occupied position also neighboring to $Y$. Then the payoff of player $c$ is $\pi_{\bar{\delta}}^{c}\left(s^{*}\right)=w\left(A_{x}\right)-w(Y)<w\left(A_{x}\right)=\pi_{\bar{\delta}}^{c}\left(y, s^{*-c}\right)$. Since she can now beneficially deviate, $s^{*}$ is not a robust equilibrium.

Furthermore, if $s^{*} \in S$ is robust, an isolated player $c \in P$ may never have an incentive to deviate to a directly or indirectly neighboring position $x^{\prime} \in \bar{X}$. Because the weight of all competitive zones surrounding $x$ equals $0, \pi^{c}\left(s^{*}\right)=w\left(H_{x}\left(s^{*}\right)\right)=w\left(A_{x}\right)$ for all perceptions of distances. Suppose $c$ relocates to $x^{\prime}$. Similar as before, it is possible to construct individual distances $\left(\bar{\delta}_{e}^{i}\right)_{e \in E}$ for all $i \in N$ such that every agent with favorite object in $A_{x^{\prime}}$ or $A_{x}$ strictly prefers $x^{\prime}$ to any other occupied position, i.e., $\pi_{\frac{\delta}{\delta}}^{c} x^{\prime}, s^{*-c}=\frac{w\left(A_{x^{\prime}}\right)+w\left(A_{x}\right)}{p_{x^{\prime}}+1}$. But this implies

$$
\begin{aligned}
\pi^{c}\left(s^{*}\right) & =w\left(H_{x}\right) \\
& \geqslant \underbrace{\frac{w\left(A_{x^{\prime}}\right)+w\left(H_{x}\right)}{p_{x^{\prime}}+1} \forall x^{\prime} \text { (indirectly) neighboring to } x .}_{\text {highest possible payoff at occupied and (indirectly) neighboring nodes }} \Longleftrightarrow w\left(H_{x}\right) \\
& \geqslant \frac{w\left(A_{x^{\prime}}\right)}{p_{x^{\prime}}} \forall x^{\prime} \text { (indirectly) neighboring to } x .
\end{aligned}
$$

Now let $p_{x} \geqslant 1$. Because $s^{*} \in S$ is supposed to be a robust equilibrium, it is not possible to perturb distances in such a way that a player can increase her payoff. This implies that the payoff she attains at least has to be greater than the highest possible gain she can reach if she deviates. With similar arguments as in the case $p_{x}=1$ this yields

$$
\underbrace{\frac{w\left(H_{x}\right)}{p_{x}}}_{\text {worst-case payoff at } x} \geqslant \underbrace{w(\widehat{Z})}_{\text {best-case payoff at unoccupied nodes }},
$$

where $\widehat{Z} \in Z$ is the heaviest unoccupied zone, and

$$
\frac{w\left(H_{x}\right)}{p_{x}} \geqslant \underbrace{\frac{w\left(A_{x^{\prime}}\right)}{p_{x^{\prime}}+1} \forall x^{\prime} \in \bar{X} \backslash\{x\}}_{\text {best-case payoff at already occupied nodes }}
$$

If $p_{x}=1$, (2) already implies (3) for directly and indirectly neighboring objects.

Sufficiency: Now assume the requirements given in the proposition are satisfied. We have to show that the strategies where $p_{x}$ players locate at $x \in \bar{X}$ constitute robust equilibria. First consider the case $p_{x}=1$, i.e., a singly occupied node. Conditions (3.) and (4.) make sure that the player cannot improve by deviating to a neighboring competitive zone or by deviating to a directly or indirectly neighboring occupied node. Condition (1.) assures that she cannot improve by deviating to any other unoccupied zone and by Condition (2.) she cannot improve by deviating to any other occupied node. Now, let $p_{x}>1$. For a player located on $x \in \bar{X}$, Condition (1.) assures that he cannot improve by deviating to any other unoccupied zone and Condition (2.) assures that he cannot improve by deviating to any other occupied node.

Proposition 1 provides the requirements for a strategy profile to be a robust equilibrium. It consists of four straightforward conditions. The first one formalizes that deviations into unoccupied zones are never beneficial. Even if the players only receive their worst-case payoff, i.e., the weight of their hinterland, they never gain from relocating into any $Z \in \mathcal{Z}$. $^{7}$ Similarly, Condition (2.) captures that deviations to already occupied nodes $x^{\prime} \in \bar{X}$ are not beneficial. The highest possible payoff a deviating player could get is $\frac{w\left(A_{X^{\prime}}\right)}{p_{x^{\prime}}+1} .8$ These two previous considerations must be strengthened When considering certain deviations of an isolated player because her node becomes unoccupied then. Again, we distinguish between deviations into a neighboring zone and deviations on occupied nodes, which is reflected by Conditions (3.) and (4.). The main intuition is that for some distance perceptions an isolated player would attract only her hinterland, but by deviating she could receive her former hinterland and, in addition, the weight of some competitive zone (Condition (3.)). By deviating on a directly or indirectly neighboring occupied node she can not only share the payoffs of the players on this node, but would also regain some share of her former hinterland (Condition (4.)). For competitive zones neighboring a singly occupied node this means that their weight must be zero. We have already seen an example where this condition is violated. In Example 1 there are several singly occupied nodes which are neighboring a non-trivial competitive zone (cf. Fig. 2). ${ }^{9}$ Thus, we can immediately conclude that the given strategy profile is not a robust equilibrium.

The main importance of Proposition 1 is that it provides a convenient tool for verifying whether a strategy profile $s \in S$ (which might be a Nash equilibrium for specific edge lengths) constitutes a robust equilibrium or not. A straightforward procedure is to (i) determine the partition $\Pi(s)$, (ii) compute the weights of the hinterlands and competitive zones, and (iii) check if the four conditions characterizing a robust equilibrium are satisfied. In particular, since such an algorithm proceeds in quadratic time, finding a robust equilibrium is computationally as complex as finding a locational equilibrium. In the remainder of this subsection we will check the robustness of some prominent results from literature. We thereby focus on the class of trees and return to cycles and more general graphs in Section 3.4.

Hotelling's main result for two players on a continuous line is that both cluster on the so-called median. This finding is driven by the fact that both players tend to the center of the line to steal agents from the other player. This is illustrated for a discrete line in Fig. 4 where we can observe the incentive to increase the hinterland by moving to the discrete analog of the median.

\footnotetext{
7 This requirement also implies that the weight of unoccupied zones can never be higher than the average payoff of the players, i.e., $w(Z) \leqslant \frac{n}{p}$ for all $Z \in \mathcal{Z}$.

${ }^{8}$ A simple implication of this requirement is that in robust equilibria the number of players on occupied nodes is roughly proportional to the weights of the hinterlands: $\frac{p_{x}}{p_{x^{\prime}}+1} \leqslant \frac{w\left(H_{x}\right)}{w\left(H_{x^{\prime}}\right)} \leqslant \frac{p_{x}+1}{p_{x}}$ for all $x, x^{\prime} \in \bar{X}$.

We say that a competitive zone $Y$ is trivial if no agent has his favorite object there, i.e., $w(Y)=0$.
} 
Definition 3 (Median). A median of a node-weighted graph $(X, E, w)$ is a node $q \in X$ that balances the node weights, i.e., $w\left(C_{1}^{q}\right) \leqslant \frac{w(X)}{2}=\frac{n}{2}$, where $C_{1}^{q} \in \mathcal{Z}$ is the heaviest component of $(X, E)-\{q\}$.

In general, a median need not exist. For example, if we consider the complete graph where all weights are equal to one, we have $w\left(C_{1}^{q}\right)=n-1>\frac{n}{2}$. Nevertheless, one can show that if $(X, E)$ is a tree, a median always exists. ${ }^{10}$

The most direct way to extend Hotelling's model to graphs is to consider trees. Although this is only a special case of our set-up, much attention has been devoted to this particular class in literature. Among others, Eiselt and Laporte (1991) examined this setting and they have shown that in the two-player case for homogeneous distances both players will locate on the median of the tree. Thus, they came to the same conclusion as Hotelling did. In fact, this result had already been established by Wendell and McKelvey (1981) in slightly different terms. In their publication the authors show that for homogeneous distances on a tree the median is always a Condorcet winner. ${ }^{11}$ Since a Condorcet winner cannot be beaten in majority voting (by definition), choosing the Condorcet winner constitutes a locational equilibrium in the twoplayer game.

Now, let us apply Proposition 1 to test whether the two-player results mentioned in the previous paragraph are robust. If both players locate on the same object, say $q \in X$, there is only one hinterland consisting of all the nodes, i.e., $\Pi(s)=\{X\}$. Therefore, only Condition (1.) of Proposition 1 applies and it simplifies to $\frac{n}{2} \geqslant w(\widehat{Z})=w\left(C_{1}^{q}\right)$, which is exactly the definition of the median. ${ }^{12}$ Now, consider the setting where the players choose different positions, say $x$ and $x^{\prime} \in X$. Eiselt and Laporte (1991) show that this is a locational equilibrium only if the positions are either neighboring or the competitive zone between them has weight 0 and, furthermore, $\frac{n}{2}=w\left(C_{1}^{x}\right)=w\left(C_{1}^{x^{\prime}}\right)$ holds. Applying conditions (3.) and (4.) of Proposition 1 yields that this is robust, too.

In Eiselt and Laporte (1993) the authors examine the case of three players on a tree. In their main result they distinguish four different cases: (i) type A equilibria (all players cluster on the median $q \in X$ ), (ii) type B equilibria (two players locate on the median $q$ and one in the heaviest component $C_{1}^{q} \in \mathcal{Z}$ on the node that is neighboring to $q$ ), (iii) type $C$ equilibria (all three players on different nodes), and (iv) non-existence of equilibria. With the conditions given in Eiselt and Laporte (1993) it is easy to check that type $A$ and type B equilibria are indeed robust. However, type C equilibria generically are not. They are robust only if the hinterland of all players has the same weight because otherwise Condition (4.) of Proposition 1 would be violated.

Note that in the previous examples the equilibria are robust only if some kind of minimal differentiation is satisfied and at least some players choose the median $q$. Therefore these results raise some questions regarding the general form of robust equilibria.

\footnotetext{
10 Moreover, for trees a node $q$ is a median if and only if $q \in \operatorname{argmin}\left\{\sum_{y \in X} d(x, y) w_{y} \mid\right.$ $x \in X\}$ (see Goldman, 1971), i.e., a median $q$ is a minimizer of the weighted sum of graph distances, which holds true for all possible edge lengths. On general graphs there are multiple conventions for the notion 'median': sometimes it is defined (rather than characterized) as the minimizer of the weighted sum of graph distances.

11 Later Hansen, Thisse, and Wendell (1986) extended this work.

12 In fact, this has already been shown for the continuous line, although in very different terms, by the seminal contribution of Black (1948). He proved that for single-peaked preferences on a line the median is always a Condorcet winner. As already mentioned in Section 2, single-peaked preferences on a line are is equivalent to our assumption of heterogeneous edge lengths on the line graph.
}

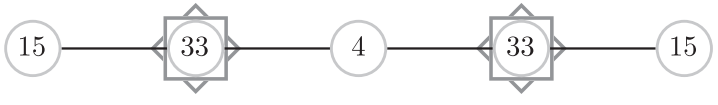

Fig. 5. A robust equilibrium with no player on the median and without minimal differentiation.

\subsection{Minimal differentiation}

Minimal differentiation is one of the most controversial results and much attention has been devoted to its validity. ${ }^{13}$ In the framework of graphs, we define minimal differentiation as follows.

Definition 4. A strategy profile $s \in S$ satisfies minimal differentiation if all players locate on the same node, i.e., $s=(x, x, \ldots, x)$ for some $x \in X$.

In the previous section there were already examples for robust equilibria satisfying minimal differentiation for two or three players. ${ }^{14}$ These cases can be extended to arbitrary numbers of players in a straightforward way. Consider the strategy profile $s:=(x, x, \ldots, x)$ where all players locate on a node $x \in X$. We then have only one hinterland consisting of all the nodes, i.e., $\Pi(s)=\{X\}$. By using the same arguments as in the two-player case one can see that Conditions (2.), (3.), and (4.) of Proposition 1 do not apply and, furthermore, Condition (1.) simplifies to $\frac{n}{p} \geqslant w(\widehat{Z})$, where $\widehat{Z}$ is the heaviest unoccupied zone. Thus, we get the following corollary.

Corollary 1. Let $(X, E, w)$ be a node-weighted graph and $q \in X$. Furthermore, let $C_{1}^{q} \in \mathcal{Z}$ be a heaviest component of $(X, E)-\{q\}$. The strategy profile $s=(q, \ldots, q)$ is a robust equilibrium if and only if the weight of any component of $(X, E)-\{q\}$ is not higher than the average payoff, i.e., $w\left(C_{1}^{q}\right) \leqslant \frac{n}{p}$.

Corollary 1 shows that it is easy to construct a robust equilibrium for any number of players. The result is also easy to prove without Proposition 1 since for $s=(q, \ldots, q)$ every player earns the average payoff $\frac{n}{p}$, while the most beneficial deviation leads to the heaviest unoccupied zone $C_{1}^{q}$. Phrased differently, if the heaviest component of the graph without $q \in X$ is relatively light, then there exists a robust equilibrium where all players locate on the same node. In particular, this also implies that $q$ has to be a median of the graph.

Note that in the robust equilibria discussed so far all players are located on or next to the median. Therefore one might suspect that in any robust equilibrium the median must be occupied (if it exists) and that the players cluster on or around it. The following example is a counter-example to this conjecture.

Example 2. Let $(X, E, w)$ be the weighted line graph depicted in Fig. 5.

Furthermore assume that two players locate on each of the nodes with weight 33 . As it is easy to check, this strategy profile is a robust equilibrium. The median, however, is the node with a weight of four and it belongs to a competitive zone. Thus, neither minimal differentiation is satisfied, nor are players located on the median.

However, consider a reduced game where we remove the two nodes to the right and we remove the two players in this area. In

\footnotetext{
13 Some works show that generically it is not satisfied (see, e.g., d'Aspremont et al., 1979; Eaton \& Lipsey, 1975; Economides, 1986) but others support it for special cases (see, e.g., de Palma et al., 1985, 1990; Hehenkamp \& Wambach, 2010). Similar considerations also apply to minimal differentiation on graphs.

14 Definition 4 captures minimal differentiation in a strong sense. A weaker version of minimal differentiation would be the requirement that there is no unoccupied node between any pair of occupied nodes or, equivalently, that there is no competitive zone.
} 
this reduced game, the unique robust equilibrium is that the remaining two players both locate on the node with 33 agents such as in the current strategy profile. Moreover, this node is the median of the reduced graph. A similar observation can be made when reducing the game by removing "the left part".

Example 2 shows that in a robust equilibrium it need not be the case that players minimally differentiate on the median. However, it seems that locally, in a kind of reduced game, this is still true. To investigate this issue, let us formally define a reduced game. Given a strategy profile $s \in S$, we define a reduced game for every occupied node $x \in \bar{X}$ by considering the objects and players in the neighboring area $A_{x} \subseteq X$. Thus, the number of players in the reduced game is $p_{x}$ and the graph is restricted to $\left(A_{x}, E_{\mid A_{x}}\right)$. For the payoffs only those agents are considered whose favorite object belongs to the neighboring area $A_{x}$ such that the node weights of the graph in the reduced game coincide to the node weights of the original game.

Corollary 2 (Reduced Games). Suppose $s^{*} \in S$ is a robust equilibrium for some $(X, E, w)$ and let $x \in \bar{X}$ be an occupied position such that $p_{x} \geqslant 2$. Then, $x$ is the median of the subgraph $\left(A_{x}, E_{\mid A_{x}}\right)$ and $(x, x, \ldots, x)$ is a robust equilibrium satisfying minimal differentiation in the corresponding reduced game.

Proof. Let $x \in \bar{X}$ be an occupied position in $s^{*} \in S$ with $p_{x} \geqslant 2$. Applying Proposition 1, Condition (1.) implies $\frac{w\left(A_{x}\right)}{p_{x}} \geqslant \frac{w\left(H_{x}\right)}{p_{x}} \geqslant w(Z)$ for every unoccupied zone surrounding $x$. But this is equivalent to the condition of Corollary $1, w\left(C_{1}^{x}\right) \geqslant \frac{w(A)}{p_{x}}$, which shows that the strategy profile $(x, \ldots, x)$ is a robust equilibrium in the reduced game. Moreover, this condition implies that the weight of the heaviest component of $\left(A_{x}, E_{\mid A_{x}}\right)-\{x\}$ is smaller than $\frac{w\left(A_{x}\right)}{2}$ which shows that $x \in \bar{X}$ is the median of the subgraph $\left(A_{x}, E_{\mid A_{X}}\right)$.

Corollary 2 shows that in any robust equilibrium a local variant of minimal differentiation is satisfied. This finding is fully in line with the "principle of local clustering" conjectured in the seminal work of Eaton and Lipsey (1975). Their principle, however, also contains the aspect that players pair, i.e., do not locate away from other firms. This aspect is also true in robust equilibria since it follows from Condition (3.) of Proposition 1 that isolated players do not neighbor a non-trivial competitive zone. This implies that singly occupied nodes must neighbor another occupied node if node weights are strictly positive. Thus, any robust equilibrium can be characterized as a few multiply occupied nodes which are possibly neighbored by some singly occupied nodes. The final question on the extent of differentiation is whether these local clusters can be at a large distance from each other.

In Example 2 only a small share of agents favor the object between the occupied positions. In fact, it holds generally that the weight of competitive zones in robust equilibria must be relatively light.

Proposition 2 (Competitive zones). Let $(X, E, w)$ be a node-weighted graph. Suppose $s^{*} \in S$ is a robust equilibrium and let $\mathcal{Y}$ be the set of competitive zones. Then, $\sum_{Y \in \mathcal{Y}} w(Y) \leqslant \frac{n}{5}$.

The proof can be found in the appendix. By definition, a strategy profile satisfies minimal differentiation only if there is no competitive zone. In this context, Proposition 2 can be interpreted as a weaker form of a global minimal differentiation result: competitive zones might exist in equilibrium, but their weight in sum is bounded by $\frac{n}{5}$, i.e., at most $20 \%$ of the agents can have their favorite object in some competitive zone.

The requirement of robustness is crucial for each of the results on minimal differentiation. Indeed, it is possible to find (nonrobust) locational equilibria which do not satisfy the properties specified by Corollaries 1 and 2, and Proposition 2. Whether robustness also leads to stronger results with respect to efficiency is addressed next.

\section{3. (In-)efficiency}

Traditionally, welfare is measured by aggregating the players' and the agents' surplus. However, from the players' perspective, in our setting (i.e., without considering price competition) any strategy profile yields the same aggregated surplus as we study a constant-sum game. Therefore, efficiency will be discussed from the viewpoint of the agents which are interpreted as consumers in this subsection. ${ }^{15}$ The standard result of two firms choosing the median of a line is known to be inefficient since minimal differentiation leads to unnecessarily high distances for the consumers. In his paper, Hotelling complains about this inefficiency:

"Buyers are confronted everywhere with an excessive sameness [...]" and "[...] competing sellers tend to become too much alike."

(Hotelling, 1929, p. 54)

This result, however, does not simply generalize. Reconsider Example 1 where some agents are uniformly distributed along a cycle graph with equal edge lengths. The (non-robust) locational equilibrium depicted in Fig. 2 is efficient with respect to different criteria. For instance, it minimizes the sum of distances (of each consumer to a closest player) as well as the sum of squared distances, which are the most common cardinal criteria. ${ }^{16}$ However, the cardinal approach does not seem to be fully justified in our context as we have individual distance perceptions which need not be comparable across consumers. A well-known ordinal criterion is Pareto efficiency. The locational equilibrium in Example 1 satisfies this criterion as well, i.e., there does not exist another strategy profile such that any consumer is at least as well off and at least one consumer is strictly better off (where better off here means that the perceived distance to the closest player becomes shorter). Note that this is a weak requirement which is satisfied by plenty of strategy profiles. The existence of locational equilibria that are efficient therefore raises the question of whether robust equilibria can be efficient as well. Under generic conditions, the answer is no.

Proposition 3 (Pareto efficiency). Let $(X, E, w)$ be a node-weighted graph. Suppose that the number of agents $n$ is not divisible by the number of players $p$ and that there are at least $p$ nodes with positive weight $w_{x}>0$. Then any robust equilibrium is Pareto dominated (for the consumers).

Proposition 3 shows that under mild conditions robust equilibria are not Pareto efficient. This statement of inefficiency with respect to an ordinal criterion precludes efficiency with respect to cardinal criteria as well since no Pareto dominated strategy profile can minimize the sum of (squared) distances. Moreover, similarly to the analysis of Gur, Saban, and Stier-Moses (2012), the price of anarchy (cf. Koutsoupias \& Papadimitriou, 2009) can be arbitrarily high in specific examples in our model. ${ }^{17}$ The proof of Proposition 3 is relegated to the appendix. Its intuition is simple.

\footnotetext{
15 These might be inhabitants that visit a facility or consumers who buy a product. Because we have not specified a second stage like government formation in our model, the discussion of efficiency does not apply to the context of voting.

16 The sum of squared distances as an efficiency criterion has been used, for example, by Król (2012) and Meagher and Zauner (2004) who find different effects of uncertainty on efficiency.

17 A different notion of "robustness" addresses the question of whether bounds for the price of anarchy with respect to Nash equilibria extend to weaker notions of equilibrium (Roughgarden, 2009). The fact that in our game it is possible to construct examples of robust equilibria with an infinite price of anarchy implies that, without additional assumptions, bounds for weaker notions of equilibrium cannot be found, either.
} 


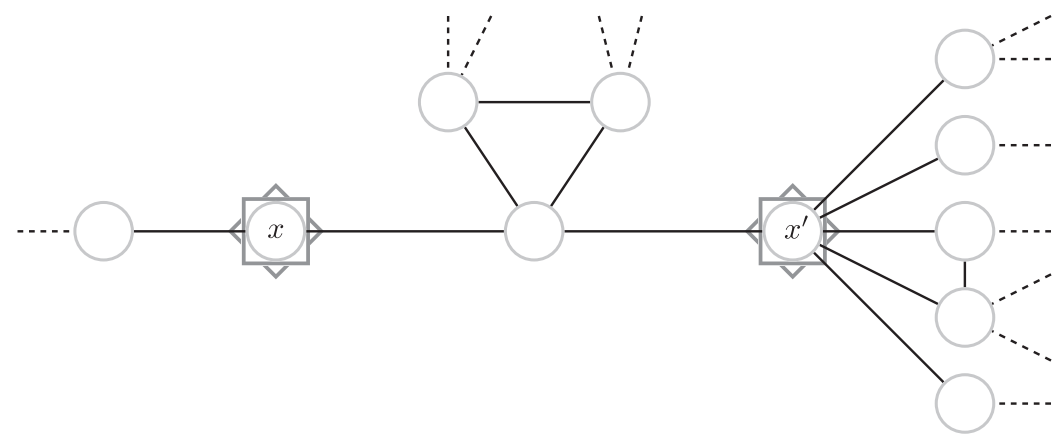

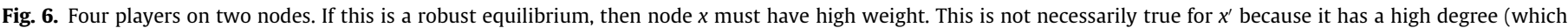
leads to several arms in its hinterland).

Generically, in every robust equilibrium there are two firms that choose the same location, while the consumers would benefit if one of them located at a different position. In fact, as we have a constant-sum game between the players, a social planner could relocate them and provide transfer payments to keep their payoffs constant. Thus, a socially optimal outcome from the consumers' point of view would be possible without changing the payoffs of the players. ${ }^{18}$ This shows that, in a much more general form, Hotelling's inefficiency persists when robustness is required.

\section{4. (Non-)existence of robust equilibria}

So far we analyzed properties of robust equilibria without explicitly examining under which conditions they exist. In Section 3.1, we have shown for small numbers of players on tree graphs that most of the sufficient conditions from the literature indeed induce robust equilibria. Moreover, Corollary 1 provides a condition which is sufficient for existence. Intuitively, it is satisfied either if the weight is concentrated on the median or if we have a star-like structure under a more equal weight distribution. Although this condition is necessary and sufficient only for robust equilibria with minimal differentiation, similar considerations also apply in general. Corollary 1 is based on Proposition 1 which characterizes the underlying strategy profiles of robust equilibria. ${ }^{19}$ In particular, Condition (1.) states that the hinterland $H_{x} \subseteq X$ of every occupied node $x \in \bar{X}$ must be heavy enough to carry $p_{x}$ players. If this weight is not directly on the node $x$, then it must be on other nodes in its hinterland. Considering the "arms" in the hinterland, i.e., the components in the graph $\left(H_{x}, E_{\mid H_{x}}\right)-\{x\}$, each of them is an unoccupied zone. However, for unoccupied zones the weight is bounded, again by Proposition 1 Condition (1.). Thus, in order to be heavy enough, an occupied node $x \in \bar{X}$ must either have sufficiently many arms in its hinterland (which are heavy in sum) or it must have a relatively high weight itself. This intuition is formalized in Corollary 3.

Corollary 3. For some node-weighted graph $(X, E, w)$, let $s^{*} \in S$ be a robust equilibrium with heaviest unoccupied zone $\widehat{Z} \in \mathcal{Z}$ (and $w(\widehat{Z})>0)$. Let $x \in \bar{X}$ be occupied by $0<p_{x}<p$ players. Denote by $a_{x} \in \mathbb{N}$ the number of arms (i.e., the number of components in the hinterland for $\left.\left(H_{x}, E_{\mid H_{x}}\right)-\{x\}\right)$ of $x$. Then

$\frac{w_{x}}{w(\widehat{Z})}+a_{x} \geqslant p_{x}$.

\footnotetext{
18 However, this result also depends on the abstraction from price competition. If firms do not cluster, i.e., if they have a local monopoly, they might have an incentive to raise prices.

19 Proposition 1 provides the necessary and sufficient conditions for existence in the sense that a robust equilibrium exists if and only if there is a strategy profile that satisfies these conditions. Thus, this result transforms the problem of finding a strategy profile that is a robust equilibrium into finding a strategy profile that satisfies the conditions of Proposition 1 , but it is not a result on the exogenously given situation of spatial competition, i.e., on the node-weighted graph $(X, E, w)$.
}

Proof. Let $\widehat{Z}_{x} \in \mathcal{Z}$ be the heaviest unoccupied zone in the hinterland of $x \in \bar{X}$. The result then follows from Proposition 1 Condition (1.):

$$
\begin{aligned}
w\left(H_{x}\right) & \geqslant p_{x} w(\widehat{Z}) \Rightarrow w_{x}+a_{x} w\left(\widehat{Z_{x}}\right) \geqslant p_{x} w(\widehat{Z}) \\
& \Rightarrow \frac{w_{x}}{w(\widehat{Z})}+a_{x} \cdot \frac{w\left(\widehat{Z_{x}}\right)}{w(\widehat{Z})} \\
& \geqslant p_{x} \Rightarrow \frac{w_{x}}{w(\widehat{Z})}+a_{x} \geqslant p_{x} .
\end{aligned}
$$

Corollary 3 shows that in a robust equilibrium the relative weight of an occupied node plus its number of arms must exceed the number of players on it. This result is illustrated in Fig. 6 with two occupied nodes $x$ and $x^{\prime} \in \bar{X}$.

While $x$ has only one arm in its hinterland, $x^{\prime}$ has four of them. Therefore, for node $x$ we have $\frac{w_{x}}{w(\widehat{Z})}+1 \geqslant 2$, which is equivalent to $w_{x} \geqslant w(\widehat{Z})$, i.e., the weight of the node must exceed the weight of the heaviest unoccupied zone. Note that this implies an inequality of weights if there are unoccupied zones with many nodes. In contrast to this, $x^{\prime}$ needs not be as heavy as $x$, but in order to have four arms it must be a cut vertex and have a degree larger than five. Thus, one interpretation for Corollary 3 is that the weight of occupied nodes and their degree can be interpreted as some kind of substitutes: at least one of them has to be high enough in order to carry $p_{x}$ players in equilibrium.

This gives a requirement for robust equilibria on the level of single nodes. On the graph level this requirement will translate into (a) structural features of the graph and in (b) conditions on the distribution of weights. To assess the weight distribution, we consider the inequality of weights measured by the variance. In our case it is given by $\operatorname{Var}(w)=\sum_{x \in X}\left(w_{x}-\frac{n}{\xi}\right)^{2}=\frac{1}{\xi} \sum_{x \in X} w_{x}^{2}-\frac{n^{2}}{\xi^{2}}$. The variance is the quadratic distance from the uniform distribution. In particular, $\operatorname{Var}(w)=0$ if and only if $w_{x}=\frac{n}{\xi}$ for all $x \in X$, i.e., if and only if $w$ is uniformly distributed (a special case that is predominantly discussed in the literature). To assess structural requirements of a graph we consider its connectedness which is measured by the number of blocks $b$ (cf. Diestel, 2005). If this number is smaller than the number of players $p$, then it is still impossible to have Equation (4) trivially satisfied (such as for node $x^{\prime}$ in Fig. 6). For these graphs Corollary 3 has implications on the weight distribution because there must be an occupied node that is similar to node $x$ in Fig. 6. As a consequence, we have that graphs with a high connectivity (i.e., a relatively small number of blocks) only admit robust equilibria if the weight distribution is far from uniform.

Proposition 4. Let $(X, E, w)$ be a node-weighted graph with $\xi>3 p$. Suppose that the number of blocks is smaller than the number of players, i.e., $b<p$. Then there exists some $v>0$ such that $\operatorname{Var}(w)<v$ implies that a robust equilibrium does not exist. 
The interpretation of this result is as follows: suppose the graph is not too small $(\xi>3 p)$ and the distribution of agents is sufficiently close to the uniform distribution. Then the existence of robust equilibria requires a low connectivity of the underlying graph in terms of that there must be more blocks than players.

Proposition 4 obviously applies to all graphs with just one block (i.e., $b=1$ ) like grids, for instance. Those graphs are known as twoconnected and they are characterized by not containing any cut vertex (see, e.g., Diestel, 2005). Indeed, in this case we have $a_{x}=0$ for any occupied node $x \in \bar{X}$ (and for any $s \in S$ ). Thus, if a two-connected graph is sufficiently large, it always satisfies the requirements of Proposition 4 and therefore it does not admit robust equilibria if the weight distribution is too close to uniformity. ${ }^{20} \mathrm{~A}$ particular example of this class of graphs are cycle graphs (as illustrated in Fig. 2) which have been studied extensively by Mavronicolas et al. (2008). Given a uniform distribution of agents (and edge lengths), the authors have shown that there always exists a Nash equilibrium for $\xi>3 p$. However, Proposition 4 immediately implies that these equilibria are not robust.

For tree graphs Proposition 4 does not apply since trees consist of many blocks. However, for this special class the number of arms is also restricted by some structural property. Since there are no cycles in a tree, each arm in any hinterland leads to a node of degree 1 , a so-called loose end. Therefore, completely analogous to Proposition 4 we can show the following.

Proposition 5. Let $(X, E)$ be a node-weighted tree with $\xi>3 p$. Suppose that $e<p$, where $e$ is the number of loose ends. Then there exists some $v>0$ such that $\operatorname{Var}(w)<v$ implies that no robust equilibrium exists.

The number of loose ends is a structural feature that is related to the equality of the degree distribution of the graph. The lowest number of loose ends in a tree is attained in the line graph (which has a highly equal degree distribution), while the highest number is attained in the star graph (which has a highly unequal degree distribution). In that sense, Proposition 5 shows that the existence of a robust equilibrium on a tree requires either an unequal distribution of weight or an unequal distribution of degree.

To sum up, robust equilibria certainly exist for structures that are similar to a star graph (Corollary 1 ) or have a highly concentrated distribution of weights. However, for graphs with few cut vertices (i.e., graphs with a low number of blocks) and for tree graphs, robust equilibria can exist only if the weight distribution is not close to uniform. To consider a numerical example for the required inequality: for trees that satisfy the condition $e<p$ of Proposition 5 and for cycle graphs (which always satisfy the condition $b<p$ of Proposition 4) we can show that there only exists a robust equilibrium of three or more players if there is a node $x \in X$ that is at least $\frac{\xi}{p}-1$ times heavier than some other node $x^{\prime} \in X$. Thus, if the number of nodes strongly exceeds the number of players in the game (i.e., $\xi \gg p$ ), those one-dimensional structures do not admit robust equilibria if the weights are uniformly distributed.

\section{Discussion}

Models of spatial competition predominantly deal with three important applications: (i) firms that strategically locate facilities (e.g., Eiselt \& Laporte, 1993), (ii) political candidates who strategi-

\footnotetext{
20 The result that two-connected graphs require a sufficient inequality of node weights can also be derived from Proposition 2. Since in two-connected graphs any unoccupied node belongs to a competitive zone, Proposition 2 implies that we have $w(\bar{X}) \geqslant \frac{4}{5} n$ in robust equilibria. Thus, there must be at least one node $x$ with $w_{x} \geqslant \frac{4}{5} \frac{n}{p}$. That is, to reach an average payoff $\frac{n}{p}$ it is almost enough to attract all agents with favorite object $x$.
}

cally choose a political platform (e.g., de Palma et al., 1990), and (iii) firms that strategically choose a product specification (e.g., Eaton \& Lipsey, 1975). In any of the model variations it has been standardly assumed that agents are heterogeneous with respect to their ideal point (i.e., location/policy/product), but homogeneous with respect to the perception of distances. In particular, it must hold that two agents with the same ideal point agree on the ranking of all the other alternatives. In this paper, we have introduced a way to relax this strong homogeneity requirement by considering individual distance perceptions. We assess whether model predictions are robust in the sense that they are independent of the perceived distances. Thereby, we confirm robustness of the equilibria found for two and three players on a tree graph by Eiselt and Laporte (1991, 1993). And we find strong support for a conjecture of the "principle of local clustering" articulated by Eaton and Lipsey (1975, p. 46) who further explain that "[t]he principle of minimum differentiation is a special case of the principle of local clustering when the number of firms in the market is restricted to two." In fact, we have shown that all robust equilibria satisfy local clustering in the sense that we have minimal differentiation in each reduced game. An implication of this result is that robust locational choices are not Pareto efficient, which is in line with Hotelling's conjecture. On the other hand, not all results from models of spatial competition are robust with respect to heterogeneous distance perceptions. Especially in graphs without cut vertices the existence of robust equilibria is highly restricted. We illustrate this in an example of uniform distribution of agents along a cycle graph (analyzed by Mavronicolas et al., 2008). Indeed, by discussing general structural conditions for the existence of robust equilibria, we have shown that the existence generically requires a highly unequal distribution of agents. This also raises the question whether there are robust outcomes in the three main applications mentioned at the beginning of this section. For example, Proposition 2 implies that at most $20 \%$ of the agents may have their favorite object "between" the players. Interestingly, some empirical data on the geographical distribution of inhabitants suggests that the necessary inequality requirements might just be satisfied. According to the United Nations report from 2012 the rate of urbanization in more developed regions was about 78\% in 2011 and it is still increasing. ${ }^{21}$ In the US it was even higher than $82 \%$, for example. Thus, the population in more developed regions is quite unequally distributed and this suggests that if firms serve only the major cities this might well be a robust equilibrium, despite the inefficiency for consumers who live outside these cities. In the case of product or policy spaces, the exact distribution of consumers is still an open question. But if it should not meet the requirements of robust equilibria, this would lead again to our main motivation that the assumption of homogeneous distances can have a strong impact on the results. In this case, the use of models of spatial competition in these applications has to be reconsidered carefully.

Although we have focused in this paper on just one - yet crucial - aspect of robustness, several other model specifications can be challenged as well. Some of them do not substantially influence our results. For instance, if the assumption that the players do not locate on the edges of the graph was relaxed, then for any robust equilibrium in this more general set-up there exists another one where the players only locate on the vertices and each of them attracts the same set of agents. Moreover, these additional equilibria exist only under very restrictive conditions. Another aspect that could be relaxed is the assumption that ties are broken equally in the case of equal distances. Although it would then be necessary to adapt the formulations of the results, their substance would not change. The reason is that robust equilibria are independent of

\footnotetext{
${ }^{21}$ United Nations, DESA (2012). World urbanization prospects: the 2011 revision.
} 
the perceived distances and, thus, the tie-breaking rule is relevant only if two players locate at the same position.

On the other hand, however, there are also further assumptions which might well play an important role. In particular, we study a simultaneous move game, while models of sequential moves lead to quite different predictions about minimal differentiation (e.g., Loertscher \& Muehlheusser, 2011; Prescott \& Visscher, 1977), when more than two players are involved. ${ }^{22} \mathrm{~A}$ further major modeling decision is whether continuous or discrete space is considered. We have contributed to bridging the two corresponding literatures, but it is left for future research to clarify the role of this modeling assumption; for instance, by approximating a continuous space by a discrete space of shrinking steps.

\section{Acknowledgements}

We are grateful to Claude d'Aspremont, Chiara Canta, Herbert Dawid, Martina Eikel, Claus-Jochen Haake, Burkhard Hehenkamp, François Maniquet, Gerd Muelheusser, Tom Truyts, Fernando Vega-Redondo, and two anonymous referees for helpful comments and suggestions. Special thanks go to Jan-Philip Gamp for many interesting and inspiring discussions. We also thank session participants at the "Fourth Congress of the Game Theory Society (GAMES 12)" in Istanbul and at the workshop "Models of Influence and Network Theory (MINT 12)" in Paris as well as seminar audiences at CORE (Louvain-la-Neuve) and FUSL (Brussels). Nils Roehl gratefully acknowledges financial support by the German Research Foundation (DFG) within the Collaborative Research Center "On-The-Fly Computing" (SFB 901).

\section{Appendix A. Proofs}

Proof of Proposition 2. Let $s^{*} \in S$ be a robust equilibrium and $\underline{x} \in \bar{X}$ be the position with lowest worst-case payoff, i.e., $\frac{w\left(H_{\underline{x}}\right)}{p_{x}} \leqslant \frac{w\left(H_{x}\right)}{p_{x}}$ for all $x \in \bar{X}$. Then Proposition 1 Condition (2.) implies

$$
\begin{aligned}
w\left(H_{\underline{x}}\right) & \geqslant \frac{p_{\underline{x}}}{p_{x}+1} w\left(A_{x}\right)=\frac{p_{\underline{x}}}{p_{x}+1}\left(w\left(H_{x}\right)+\sum_{Y \in \mathcal{Y}, Y \subseteq A_{x}} w(Y)\right) \\
& \geqslant \frac{p_{x}}{p_{x}+1} w\left(H_{\underline{x}}\right)+\frac{p_{\underline{x}}}{p_{x}+1} \underbrace{\sum_{Y \in \mathcal{Y}, Y \subseteq A_{x}} w(Y)}_{=: w\left(\mathcal{Y}_{x}\right)}
\end{aligned}
$$

and, consequently, $w\left(H_{\underline{x}}\right) \geqslant p_{\underline{x}} w\left(\mathcal{Y}_{x}\right)$ for all $x \in \bar{X} \backslash\{\underline{x}\}$, where $w\left(\mathcal{Y}_{x}\right)$ is the aggregated weight of competitive zones surrounding $x \in \bar{X}$.

Case 1: $p_{x}=1$.

Here, Proposition 1 Condition (3.) implies $w(Y)=0$ for all $Y \subseteq A_{x}$ and, thus, $w\left(H_{\underline{x}}\right) \geqslant p_{\underline{x}} w\left(\mathcal{Y}_{\underline{x}}\right)=0$. Then:

$$
\begin{aligned}
n & =\sum_{x \in \bar{X}} w\left(H_{x}\right)+\sum_{Y \in \mathcal{Y}} w(Y) \geqslant \sum_{x \in \bar{X}} p_{x} \cdot \frac{w\left(H_{\underline{x}}\right)}{p_{\underline{x}}}+\sum_{Y \in \mathcal{Y}} w(Y) \\
& \geqslant \sum_{x \in \bar{X}} p_{x} \underbrace{w\left(\mathcal{Y}_{x}\right)}_{=0, \text { if } p_{x}=1}+\sum_{Y \in \mathcal{Y}} w(Y) \geqslant 2 \sum_{x \in \bar{X}} w\left(\mathcal{Y}_{x}\right)+\sum_{Y \in \mathcal{Y}} w(Y) \\
& \geqslant 2\left(2 \sum_{Y \in \mathcal{Y}} w(Y)\right)+\sum_{Y \in \mathcal{Y}} w(Y)=5 \sum_{Y \in \mathcal{Y}} w(Y),
\end{aligned}
$$

where the last inequality is due to the fact that by definition of competitive zones each $Y \in \mathcal{Y}$ is neighboring to at least two occupied positions.

Case 2: $p_{\underline{x}} \geqslant 2$.

\footnotetext{
22 Also in the literature on sequential location choices the questionable homogeneity assumption is standard. When relaxing this assumption one can find simple three-player examples where the equilibria are not robust.
}

If $p_{x}=1$ for all $x \in \bar{X} \backslash\{\underline{x}\}$, again Condition (3.) from Proposition 1 implies $w\left(\mathcal{Y}_{x}\right)=0$ for all $x \in \bar{X} \backslash\{\underline{x}\}$ and there remains nothing to show. Therefore, assume that there exists at least one $x^{\prime} \in \bar{X} \backslash\{\underline{x}\}$ with $p_{x^{\prime}} \geqslant 2$. Again, one can exploit Proposition 1 Condition (2.):

$$
\begin{aligned}
w\left(H_{\underline{x}}\right) & \geqslant \frac{p_{\underline{x}}}{p_{x^{\prime}}+1} w\left(A_{x^{\prime}}\right) \Longleftrightarrow w\left(H_{\underline{x}}\right) \\
& \geqslant p_{\underline{x}} w\left(A_{x^{\prime}}\right)-p_{x^{\prime}} w\left(H_{\underline{x}}\right) \Longleftrightarrow w\left(H_{\underline{x}}\right) \\
& \geqslant p_{\underline{x}} w\left(H_{x^{\prime}}\right)-p_{x^{\prime}} w\left(H_{\underline{x}}\right)+p_{\underline{x}} w\left(\mathcal{Y}_{x^{\prime}}\right)
\end{aligned}
$$

and, analogously,

$$
\begin{aligned}
w\left(H_{x^{\prime}}\right) & \geqslant \frac{p_{x^{\prime}}}{p_{\underline{x}}+1} w\left(A_{\underline{x}}\right) \Longleftrightarrow w\left(H_{x^{\prime}}\right) \\
& \geqslant p_{x^{\prime}} w\left(H_{\underline{x}}\right)-p_{\underline{x}} w\left(H_{x^{\prime}}\right)+p_{x^{\prime}} w\left(\mathcal{Y}_{\underline{x}}\right) .
\end{aligned}
$$

Now, the rest of the proof proceeds similarly to Case 1. According to (1) we can again decompose the graph in hinterlands and competitive zones and by using $w\left(H_{\underline{x}}\right) \geqslant p_{\underline{x}} w\left(\mathcal{Y}_{x}\right)$ for all $x \in \bar{X} \backslash\{\underline{x}\}$ one gets

$$
\begin{aligned}
n= & w\left(H_{\underline{x}}\right)+w\left(H_{x^{\prime}}\right)+\sum_{x \in \bar{X} \backslash\left\{\underline{\underline{x}}, x^{\prime}\right\}} w\left(H_{x}\right)+\sum_{Y \in \mathcal{Y}} w(Y) \\
\geqslant & p_{\underline{\underline{x}}} w\left(H_{x^{\prime}}\right)-p_{x^{\prime}} w\left(H_{\underline{x}}\right)+p_{\underline{x}} w\left(\mathcal{Y}_{x^{\prime}}\right)+p_{x^{\prime}} w\left(H_{\underline{x}}\right)-p_{\underline{x}} w\left(H_{x^{\prime}}\right) \\
& +p_{x^{\prime}} w\left(\mathcal{Y}_{\underline{x}}\right)+\sum_{x \in \bar{X} \backslash\left\{\underline{x}, x^{\prime}\right\}} p_{x} \cdot \frac{w\left(H_{\underline{x}}\right)}{p_{\underline{x}}}+\sum_{Y \in \mathcal{Y}} w(Y) \\
\geqslant & p_{\underline{\underline{x}}} w\left(\mathcal{Y}_{x^{\prime}}\right)+p_{x^{\prime}} w\left(\mathcal{Y}_{\underline{x}}\right)+\sum_{x \in \bar{X} \backslash\left\{\underline{\underline{x}}, x^{\prime}\right\}} p_{x} \underbrace{w\left(\mathcal{Y}_{x}\right)}_{=0, \text { if } p_{x}=1}+\sum_{Y \in \mathcal{Y}} w(Y) \\
\geqslant & 2 \sum_{x \in \bar{X}} w\left(\mathcal{Y}_{x}\right)+\sum_{Y \in \mathcal{Y}} w(Y) \geqslant 2\left(2 \sum_{Y \in \mathcal{Y}} w(Y)\right)+\sum_{Y \in \mathcal{Y}} w(Y) \\
= & 5 \sum_{Y \in \mathcal{Y}} w(Y) .
\end{aligned}
$$

Again, the last inequality holds because each $Y \in \mathcal{Y}$ is neighboring to at least two occupied positions.

Proof of Proposition 3. Let $(X, E, w)$ be a node-weighted graph and suppose $\frac{n}{p} \notin \mathbb{N}$. We first show that in any robust equilibrium $s^{*} \in S$ there is at least one node multiply occupied.

Suppose the opposite is true: there is a robust equilibrium $s \in S$ with only singly occupied nodes, i.e., $p_{x}=1$ for all $x \in \bar{X}$. Consider two occupied nodes $x, x^{\prime} \in \bar{X}$ which are directly or indirectly neighboring. Condition (4.) of Proposition 1 then reads $w\left(H_{X}\right) \geqslant \frac{w\left(A_{X^{\prime}}\right)}{1} \geqslant \frac{w\left(H_{X^{\prime}}\right)}{1}$ and $w\left(H_{X^{\prime}}\right) \geqslant \frac{w\left(A_{X}\right)}{1} \geqslant \frac{w\left(H_{X}\right)}{1}$ which implies that $w\left(H_{X}\right)=w\left(H_{X^{\prime}}\right)$. Since the graph $(X, E)$ is connected, any occupied node $x \in \bar{X}$ is a direct or indirect neighbor of at least one other occupied node and the relation of being a (direct or indirect) neighbor connects all occupied nodes. Therefore, we have $w\left(H_{x}\right)=w\left(H_{x^{\prime}}\right)$ for all $x, x^{\prime} \in \bar{X}$. Moreover, Condition (3.) of Proposition 1 implies that all competitive zones must have a weight of zero (because they have a singly occupied node as a neighbor) such that $\sum_{x \in \bar{X}} w\left(H_{x}\right)=n$. Taken together, this yields $w\left(H_{x}\right)=\frac{n}{p}$ for any $x \in \bar{X}$. However, since the weight of each hinterland is determined by a number of agents, we must have $w\left(H_{x}\right) \in \mathbb{N}$, which contradicts our assumption that $\frac{n}{p} \notin \mathbb{N}$.

Thus, in every robust equilibrium there needs to be a multiply occupied node, say $x \in \bar{X}$. Since at least $p$ nodes have a positive weight, there exists an unoccupied node, say $\tilde{x} \in X \backslash \bar{X}$, with $w_{\tilde{x}}>0$. Changing the strategy of one player with $s^{c}=x$ to $\tilde{s}^{c}=\tilde{x}$ is a Pareto improvement because all consumers with $\hat{x}^{i}=\tilde{x}$ are better off. 
Proof of Proposition 4. To show the proposition, assume the opposite is true: that is, assume there exists a robust equilibrium $s^{*} \in S$. Let $\widehat{Z} \in \mathcal{Z}$ be the heaviest unoccupied zone with respect to $s^{*}$. Given the requirements of the proposition, we will show that in each robust equilibrium there exists an occupied node which is heavier than $\widehat{Z}$. If the variance becomes small, this leads to a contradiction. The proof proceeds in five steps:

Step 1: The $\epsilon-v_{\epsilon}$-criterion.

Consider the mapping $\|\cdot\|_{1}: \mathbb{R}^{\xi} \rightarrow \mathbb{R}$ with $\|w\|_{1}=\sum_{x \in X}\left|w_{x}\right|$ also known as the Manhattan norm. It is well-know that $\|\cdot\|_{1}$ is continuous. Thus, for all $\epsilon>0$ there exists some $v_{\epsilon}>0$ such that $\left\|w-w^{\prime}\right\|_{2}<v_{\epsilon}$ implies $\left\|w-w^{\prime}\right\|_{1}<\epsilon$ for all $w, w^{\prime} \in \mathbb{R}^{\xi}$, where $\left\|w-w^{\prime}\right\|_{2}=\sqrt{\sum_{x \in X}\left(w_{x}-w_{x}^{\prime}\right)^{2}}$ is, as usual, the Euclidean norm. Let $\epsilon:=\frac{2 p}{5(p+1)} \cdot \frac{n}{\xi}$. Furthermore, in the following let $w^{\prime}$ be the uniform distribution $w_{x}^{\prime}:=\frac{n}{\xi}$ for all $x \in X .^{23}$ Having specified these variables, the $\epsilon-v_{\epsilon^{-}}$ criterion from above implies that there exists some $v:=v_{\epsilon}^{2}>0$ such that from $\sqrt{\operatorname{Var}(w)}=\left\|w-w^{\prime}\right\|_{2}<\sqrt{v}$ always $\sum_{x \in X}\left|w_{x}-\frac{n}{\xi}\right|<\epsilon=\frac{2 p}{5(p+1)} \cdot \frac{n}{\xi}$ follows. Correspondingly, for the rest of the proof it is assumed that there is given a tupel of node weights $\left(w_{x}\right)_{x \in X}$ (i.e., $w \geqslant 0$ and $\sum_{x \in X} w_{x}=n$ ) with $\operatorname{Var}(w)<v$.

Step 2: We establish that $|w(\widehat{X})-| \widehat{X}\left|\frac{n}{\xi}\right|<\epsilon$ for all $\widehat{X} \subseteq X$. If $\operatorname{Var}(w)<v$, Step 1 implies for all subsets $\widehat{X} \subseteq X$, $|w(\widehat{X})-| \widehat{X}\left|\frac{n}{\xi}\right|=\left|\sum_{x \in \widehat{X}}\left(w_{x}-\frac{n}{\xi}\right)\right| \leqslant \sum_{x \in \widehat{X}}\left|w_{x}-\frac{n}{\xi}\right| \leqslant \sum_{x \in X}$ $\left|w_{x}-\frac{n}{\xi}\right|<\epsilon$

Step 3: We establish that $\sum_{x \in \bar{X}} a_{x} \leqslant b$.

The main intuition of this step is that all unoccupied zones can be covered by blocks of the graph and we will show that minimal covers of different zones have to be disjoint. Let $Z_{x} \neq Z_{X^{\prime}}$ be two unoccupied zones in the hinterland of $x$ and $x^{\prime}$, respectively, where $x, x^{\prime} \in \bar{X}$. Note that $x=x^{\prime}$ is allowed but, nevertheless, the two zones may not be equal. If it is not possible to find such two zones, $\sum_{x \in \bar{X}} a_{x} \leqslant 1$ and there remains nothing to show. According to Section 2, let $\mathcal{B}$ be the set of blocks. Obviously, $X=\bigcup_{B \in \mathcal{B}} B$ holds. Therefore there exist $\mathcal{B}^{Z_{x}}, \mathcal{B}^{Z_{x^{\prime}}} \subseteq \mathcal{B}$ with $Z_{x} \subseteq \bigcup_{B \in \mathcal{B}^{Z_{x}}} B$ and $Z_{x^{\prime}} \subseteq \bigcup_{B \in \mathcal{B}^{z^{\prime}}} B$ such that both sets are minimal with respect to inclusion, i.e., $\widehat{\mathcal{B}} \subsetneq \mathcal{B}^{Z_{x}}$ implies $Z_{x} \nsubseteq \bigcup_{B \in \widehat{\mathcal{B}}} B$ (analogously for $\widehat{\mathcal{B}} \subsetneq \mathcal{B}^{Z_{x^{\prime}}}$ ). Given the construction of blocks, the two sets $\mathcal{B}^{Z_{x}}$ and $\mathcal{B}^{Z_{x^{\prime}}}$ must be disjoint because otherwise there would be a path from $Z_{x}$ to $Z_{x^{\prime}}^{\prime}$ not passing through $x$ and $x^{\prime}$, which is not possible due to the definition of hinterlands. Thus:

$$
\sum_{x \in \bar{X}} a_{x}=\sum_{x \in \bar{X}} \sum_{Z_{x} \in \mathcal{Z}, Z_{x} \subseteq H_{x}} 1 \leqslant \sum_{x \in \bar{X} Z_{x} \in \mathcal{Z}, Z_{x} \subseteq H_{x}}\left|\mathcal{B}^{Z_{x}}\right| \leqslant|\mathcal{B}|=b .
$$

Step 4: We establish that $w_{x^{\prime}} \geqslant w(\widehat{Z})$ for some $x^{\prime} \in \bar{X}$. As already has been shown in Step 3, the number of hinterlands is bounded by $b$ and, thus, $\sum_{x \in \bar{X}} a_{x} \leqslant b<p=\sum_{x \in \bar{X}} p_{x}$. Therefore, there exists some $x^{\prime} \in \bar{X}$ with $a_{x^{\prime}} \leqslant p_{x^{\prime}}-1$ and by applying Corollary 3 this yields $w_{x} \geqslant w(\widehat{Z})$. In words: there necessarily exists an occupied node which is heavier than the heaviest unoccupied zone.

\footnotetext{
$\overline{23}$ Because the fraction $\frac{n}{\xi}$ need not be an integer, the uniform distribution cannot always be induced by allocating $n$ agents to nodes. Still, it is possible to study the node-weighted graph $\left(X, E, w^{\prime}\right)$.
}

Step 5: The final contradiction.

Since the number of hinterlands is smaller than the number of players and because of Proposition 2, the average weight of unoccupied zones in hinterlands needs to be relatively high:

$$
w(\widehat{Z}) \geqslant \underbrace{\frac{\sum_{x \in \bar{X}} w\left(H_{x}\right)-w(\bar{X})}{\sum_{x \in \bar{X}} a_{x}}}_{\text {average weight of unoccupied zones in hinterlands }}>\frac{\frac{4}{5} n-w(\bar{X})}{p} .
$$

Moreover, according to Step 4 this implies that $x^{\prime}$ must be relatively heavy as well, $w_{x^{\prime}}>\frac{5^{n} n-w(\bar{X})}{p}$. But then from Step 2 it follows that

$$
\frac{n}{\xi}+\epsilon>\frac{\frac{4}{5} \xi \frac{n}{\xi}-\left(|\bar{X}| \frac{n}{\xi}+\epsilon\right)}{p} \geqslant \frac{\frac{12}{5} p \frac{n}{\xi}-p \frac{n}{\xi}-\epsilon}{p}=\frac{7 n}{5 \xi}-\frac{\epsilon}{p},
$$

which contradicts $\epsilon=\frac{2 p}{5(p+1)} \cdot \frac{n}{\xi}$. Therefore, $s^{*}$ cannot be a robust equilibrium.

\section{Appendix B. "Dummy nodes"}

To additionally motivate heterogeneous distances, we briefly discuss a model variation that is based on homogeneous distances. Indeed, it is possible to represent any situation covered by heterogeneous distances by using additional nodes ("dummy nodes") together with homogeneous distances. However, it turns out that then the fundamental relation between the objects, i.e., the spatial structure of the model, might become completely irrelevant.

To formalize this claim, recall that in our model a graph $(X, E)$ consists of a (finite) set of nodes (objects) $X$ which find an interpretation as geographical locations, political platforms, or product specifications; and of a set of links $E \subseteq X \times X$, which represent the basic relations between the objects. Now, consider a set of additional nodes ("dummy nodes") $A$ and a set of additional links $\bar{E} \subseteq A \times(X \cup A)$ that do not have these interpretations, but only serve to make the model more flexible. That is, the preferences of each agent $i \in N$ are defined over the set of objects $X$ only, but not over the dummy nodes $A$. The extended graph is then given by $\left(X_{A}, E_{A}\right):=(X \cup A, E \cup \bar{E})$. Consider, furthermore, homogeneously determined edge lengths $\left(\delta_{e}\right)_{e \in E}>0$ and $\left(\delta_{e}\right)_{e \in \bar{E}}>0$. We say that the triple $\left(X_{A}, E_{A}\right),\left(\delta_{e}\right)_{e \in E},\left(\delta_{e}\right)_{e \in \bar{E}}$ represents the agents' preferences $\left(\succeq^{i}\right)_{i \in N}$ if there are node weights $\left(w_{x}\right)_{x \in X \cup A}$ (i.e., a distribution of the agents' favorite objects) such that $d^{i}(x) \leqslant d^{i}(y)$ if and only if $x \succeq^{i} y$ for all $x, y \in X$ and $i \in N$ (analogously to Section 2.2).

Proposition B.1. Let an underlying graph $(X, E)$ and $\epsilon \in \mathbb{R}_{>0}$ be given. Suppose there is a set of agents $N$ such that each agent $i \in N$ has complete and transitive preferences $\succeq^{i}$ over the elements in $X$. Then there exists a set of dummy nodes $A$, an extended graph $\left(X_{A}, E_{A}\right)$, and (homogeneous) edge lengths $\left(\delta_{e}\right)_{e \in \bar{E}}$ such that $\left(X_{A}, E_{A}\right),\left(\delta_{e}\right)_{e \in E},\left(\delta_{e}\right)_{e \in \bar{E}}$ represents the agents' preferences for any tuple of edge lengths $\left(\delta_{e}\right)_{e \in E}$ with $\delta_{e}>\epsilon$ for all $e \in E$.

Proof. Define $A:=\left\{a_{i} \mid i \in N\right\}$ and $\bar{E}:=X \times A$. For all $x a \in X \times A$ choose $\delta_{x a}$ such that $\frac{\epsilon}{2}<\delta_{x a}<\epsilon$; and $\delta_{x a_{i}} \leqslant \delta_{x^{\prime} a_{i}}$ if and only if $x \succeq^{i} \chi^{\prime}$ for all $x, x^{\prime} \in X$ and $i \in N$. Moreover, for each agent $i \in N$, let $a_{i} \in A$ be his favorite object. Since by construction the direct link between any two nodes $x \in X$ and $a \in A$ is always the shortest path between them, it can be checked easily that $\left(\left(X_{A}, E_{A}\right),\left(\delta_{e}\right)_{e \in E},\left(\delta_{e}\right)_{e \in \bar{E}}\right)$ indeed represents the agents' preferences for any tuple of edge lengths $\left(\delta_{e}\right)_{e \in E}$ with $\delta_{e}>\epsilon$ for all $e \in E$.

Proposition B.1 shows that it is possible to cover each and every profile of preferences by means of homogeneous distances. For each agent, we can simply add a dummy node and construct links between this node and every object which become "his" shortest 
paths. The underlying graph $(X, E)$ - capturing the basic relations between geographical locations, respectively, political platforms or product specifications - is then fully irrelevant since no shortest path uses those links (given that the edge lengths do not converge to zero). Thus, allowing for dummy nodes induces a high degree of arbitrariness and the model loses its explanatory power. Other ways of introducing dummy nodes, e.g. constructing only tree graphs, somewhat mitigate this issue but can be shown to restrict generality.

\section{References}

Berman, O., \& Gavious, A. (2007). Location of terror response facilities: A game between state and terrorist. European Journal of Operational Research, 177(2) 1113-1133.

Black, D. (1948). On the rationale of group decision-making. Journal of Political Economy, 56(1), 23-34.

Braid, R. M. (2005). The equilibrium locations of three stores with different selections of differentiated products. Economics Letters, 93, 31-36.

Carpenter, G. S. (1989). Perceptual position and competitive brand strategy in a two-dimensional two-brand market. Management Science, 35(9), 1029-1044.

d'Aspremont, C., Gabszewicz, J. J., \& Thisse, J.-F. (1979). On Hotelling's stability in competition. Econometrica, 47(5), 1145-1150.

Demange, G. (1982). Single-peaked orders on a tree. Mathematical Social Sciences, 3(4), 389-396.

Denzau, A., Kats, A., \& Slutsky, S. (1985). Multi-agent equilibria with market share and ranking objectives. Social Choice and Welfare, 2(2), 95-117.

de Palma, A., Ginsburgh, V., Papageorgiou, V. V., \& Thisse, J.-F. (1985). The principle of minimum differentiation holds under sufficient heterogeneity. Econometrica, 53(4), 767-781.

de Palma, A., Hong, G.-S., \& Thisse, J.-F. (1990). Equilibria in multi-party competition under uncertainty. Social Choice and Welfare, 7(3), 247-259.

Diestel, R. (2005). Graph theory (graduate texts in mathematics). Springer.

Downs, A. (1957). An economic theory of political action in a democracy. Journal of Political Economy, 65(2), 135-150.

Eaton, B. C., \& Lipsey, R. G. (1975). The principle of minimum differentiation reconsidered: Some new developments in the theory of spatial competition. The Review of Economic Studies, 42(1), 27-49.

Economides, N. (1986). Minimal and maximal product differentiation in Hotelling's duopoly. Economics Letters, 21(1), 67-71.

Eiselt, H. A. (1992). Hotelling's duopoly on a tree. Annals of Operations Research, 40(1), 195-207.

Eiselt, H. A., \& Laporte, G. (1991). Locational equilibrium of two facilities on a tree. RAIRO, 25(1), 5-18.

Eiselt, H. A., \& Laporte, G. (1993). The existence of equilibria in the 3-facility Hotelling model in a tree. Transportation Science, 27(1), 39-43.

Eiselt, H. A., \& Laporte, G. (1997). Sequential location problems. European Journal of Operational Research, 96(2), 217-231.

Eiselt, H. A., Laporte, G., \& Thisse, J. F. (1993). Competitive location models: A framework and bibliography. Transportation, 27(1), 44-54.

Eiselt, H. A., \& Marianov, V. (Eds.). (2011). Foundations of location analysis. New York: Springer.

Gabszewicz, J. J., \& Thisse, J.-F. (1992). Location. In R. Aumann \& S. Hart (Eds.), Handbook of game theory with economic applications. Handbook of game theory with economic applications (Vols. 1, 9, pp. 281-304). Elsevier.

Godinho, P., \& Dias, J. (2010). A two-player competitive discrete location model with simultaneous decisions. European Journal of Operational Research, 207(3), 1419-1432.
Goldman, A. J. (1971). Optimal center location in simple networks. Transportation Science, 5(2), 212-221.

Gur, Y., Saban, D., \& Stier-Moses, N. E. (2012). The competitive facility location problem in a duopoly, mimeo.

Hansen, P., Thisse, J. F., \& Wendell, R. E. (1986). Equivalence of solutions to network location problems. Mathematics of Operations Research, 11, 672-678.

Hehenkamp, B., \& Wambach, A. (2010). Survival at the center - The stability of minimum differentiation. Journal of Economic Behavior \& Organization, 76(3), 853-858.

Hotelling, H. (1929). Stability in competition. The Economic Journal, 39(153), 41-57.

Jiang, A. X., Leyton-Brown, K., \& Bhat, N. A. (2011). Action-graph games. Games and Economic Behavior, 71(1), 141-173.

Koutsoupias, E., \& Papadimitriou, C. H. (2009). Worst-case equilibria. Computer Science Review, 3(2), 65-69.

Kress, D., \& Pesch, E. (2012). Sequential competitive location on networks. European Journal of Operational Research, 217(3), 483-499.

Król, M. (2012). Product differentiation decisions under ambiguous consumer demand and pessimistic expectations. International Journal of Industrial Organization, 30(6), 593-604.

Labbé, M., \& Hakimi, S. L. (1991). Market and locational equilibrium for two competitors. Operations Research, 39(5), 749-756.

Lancaster, K. (1990). The economics of product variety: A survey. Marketing Science, 9(3), 189-206.

Loertscher, S., \& Muehlheusser, G. (2011). Sequential location games. RAND Journal of Economics, 42(4), 639-663.

Mavronicolas, M., Monien, B., Papadopoulou, V. G., \& Schoppmann, F. (2008). Voronoi games on cycle graphs. In E. Ochmański \& J. Tyszkiewicz (Eds.), Proceedings of the 33rd international symposium on mathematical foundations of computer science (MFCS'08) (vol. 5162). LNCS.

Meagher, K. J., \& Zauner, K. G. (2004). Product differentiation and location decisions under demand uncertainty. Journal of Economic Theory, 117(2), 201-216.

Meagher, K. J., \& Zauner, K. G. (2005). Location-then-price competition with uncertain consumer tastes. Economic Theory, 25(4), 799-818.

Moulin, H. (1980). On strategy-proofness and single peakedness. Public Choice, 35(4), 437-455.

Mueller, D. C. (2003). Public choice III. Cambridge University Press.

Nilssen, T. (1997). Sequential location when transportation costs are asymmetric. Economics Letters, 54(2), 191-201.

Osborne, M. J. (1995). Spatial models of political competition under plurality rule: A survey of some explanations of the number of candidates and the positions they take. Canadian Journal of Economics, 28(2), 261-301.

Plastria, F. (2001). Static competitive facility location: An overview of optimisation approaches. European Journal of Operational Research, 129(3), 461-470.

Prescott, E. C., \& Visscher, M. (1977). Sequential location among firms with foresight. Bell Journal of Economics, 8(2), 378-393.

ReVelle, C. S., \& Eiselt, H. A. (2005). Location analysis: A synthesis and survey. European Journal of Operational Research, 165(1), 1-19.

Rhee, B.-D. (1996). Consumer heterogeneity and strategic quality decisions. Management Science, 42(2), 157-172.

Roemer, J. E. (2001). Political competition: Theory and applications. Harvard University Press.

Roughgarden, T. (2009). Intrinsic robustness of the price of anarchy. In Proceedings of the forty-first annual ACM symposium on theory of computing, STOC '09 (pp. 513-522). New York, NY, USA: ACM.

Salop, S. C. (1979). Monopolistic competition with outside goods. The Bell Journal of Economics, 10(1), 141-156.

Shiode, S., \& Drezner, Z. (2003). A competitive facility location problem on a tree network with stochastic weights. European Journal of Operational Research, 149(1), 47-52.

Wendell, R. E., \& McKelvey, R. D. (1981). New perspectives in competitive location theory. European Journal of Operational Research, 6(2), 174-182. 